\title{
Energy Relaxation In Collisions Of Hydrogen And Deuterium With Oxygen Atoms
}

\section{Citation}

Zhang, Peng, Vasili A. Kharchenko, M. J. Jamieson, and Alexander Dalgarno. 2009. Energy relaxation in collisions of hydrogen and deuterium with oxygen atoms. Journal of Geophysical Research 114: A0710.

\section{Published Version}

doi:10.1029/2009JA014055

\section{Permanent link}

http://nrs.harvard.edu/urn-3:HUL.InstRepos:8919557

\section{Terms of Use}

This article was downloaded from Harvard University's DASH repository, and is made available under the terms and conditions applicable to Other Posted Material, as set forth at http:// nrs.harvard.edu/urn-3:HUL.InstRepos:dash.current.terms-of-use\#LAA

\section{Share Your Story}

The Harvard community has made this article openly available.

Please share how this access benefits you. Submit a story.

\section{Accessibility}




\title{
Energy relaxation in collisions of hydrogen and deuterium with oxygen atoms
}

\author{
P. Zhang, ${ }^{1}$ V. Kharchenko, ${ }^{1,2}$ M. J. Jamieson, ${ }^{3}$ and A. Dalgarno ${ }^{1}$
}

Received 6 January 2009; revised 12 March 2009; accepted 24 March 2009; published 9 July 2009.

[1] Collision energy transfer processes between hydrogen, deuterium, and oxygen atoms in the upper atmospheres of the terrestrial planets are studied. A new set of interaction potentials has been constructed using an accurate ab initio method. Full orientationdependent scattering cross sections have been obtained quantum mechanically and have been incorporated into the construction of the linear Boltzmann kinetic equation describing the energy relaxation process. The isotope and temperature dependence of the energy relaxation parameters have been analyzed. Distributions of the secondary energetic recoil atoms have been computed and the fractions of hot atoms capable of escaping from the atmospheres of the terrestrial planets have been determined. For applications to atmospheric physics and astrophysics, we have computed effective hard sphere cross sections for $\mathrm{O}+\mathrm{H}$ and $\mathrm{O}+\mathrm{D}$ collisions that closely reproduce the energy relaxation kinetics obtained from the linear Boltzmann equation. These effective cross sections, which are functions of the laboratory frame collisional energy and the temperature of the bath gas, may be used in simulations of the thermalization of hot $\mathrm{O}, \mathrm{H}$, and $\mathrm{D}$ atoms and their escape from planets.

Citation: Zhang, P., V. Kharchenko, M. J. Jamieson, and A. Dalgarno (2009), Energy relaxation in collisions of hydrogen and deuterium with oxygen atoms, J. Geophys. Res., 114, A07101, doi:10.1029/2009JA014055.

\section{Introduction}

[2] The upper atmospheres of the terrestrial planets consist mostly of atoms of oxygen, helium and hydrogen. Collisions among them influence strongly the evolution of the planetary gases. Collisions of oxygen atoms with hydrogen and deuterium are crucial in the study of the escape processes of oxygen and hydrogen from Mars, Venus, and Earth and the isotopic evolution of the planetary light elements [Cooper et al., 1984; Gurwell and Yung, 1993; McElroy et al., 1982; Hodges, 1993; Shizgal and Arkos, 1996; Shizgal, 1998, 1999; Krest'yanikovam and Shematovich, 2005; Jamieson et al., 2006]. Significant efforts have been made to calculate the total and differential elastic-scattering cross sections, but there are differences among the reported results, which cause a large uncertainty in the nonthermal loss rate of atomic oxygen.

[3] In the present article we obtain improved potential energy curves of the $\mathrm{OH}$ molecule from which we determine the elastic differential and energy transfer scattering cross sections for collisions of oxygen atoms with hydrogen

\footnotetext{
${ }^{1}$ Institute for Theoretical Atomic, Molecular and Optical Physics, Harvard-Smithsonian Center for Astrophysics, Cambridge, Massachusetts, USA.

${ }^{2}$ Department of Physics, University of Connecticut, Storrs, Connecticut, USA. UK.

${ }^{3}$ Department of Computing Science, University of Glasgow, Glasgow,

Copyright 2009 by the American Geophysical Union. 0148-0227/09/2009JA014055\$09.00
}

and deuterium. The differential scattering cross sections are required for an accurate calculation of the collision frequency, the energy loss rate and the distributions of the secondary hot recoil atoms produced in energetic collisions which are important in considering the escape of atoms from the terrestrial planets. We obtain the energy distributions of nonthermal atoms by solving the linear Boltzmann equation on the basis of the calculated kernel, $B\left(E \mid E^{\prime}\right)$, that describes the rate of energy transfer $E \rightarrow E^{\prime}$ collisions. We construct effective hard-sphere cross sections, which accurately describe the energy relaxation processes and may be used in applications. We have also studied the energy distribution of the recoil atoms, which may escape through collision ejection.

\section{Theory}

[4] Atom-atom elastic-scattering theory has been well documented in the literature. We briefly outline here the major steps in the calculations of differential and total cross sections. The differential cross section for scattering atoms with collision energy $\varepsilon$ through the polar angle $\chi$ into element $d \Omega$ of solid angle is given by Mott and Massey [1965]

$$
\frac{d \sigma(\varepsilon, \chi)}{d \Omega}=\left|\frac{1}{k} \sum_{l=0}^{\infty}(2 l+1) \sin \eta_{l} e^{i \eta_{l}} P_{l}(\cos \chi)\right|^{2},
$$

where $k^{2}=2 \mu \varepsilon / \hbar^{2}$ is the wave number, $\mu$ is the reduced mass, $\hbar$ is Planck's constant divided by $2 \pi, \eta_{l}$ is the phase 
shift of the $l$ 'th partial wave and $P_{l}$ denotes the Legendre polynomial. The radial wave function is $R^{-1} u_{l}(R)$, where $u_{l}(R)$ satisfies the differential equation

$$
\left[\frac{d^{2}}{d R^{2}}-\frac{l(l+1)}{R^{2}}+k^{2}-\left(\frac{2 \mu}{\hbar^{2}}\right) V(R)\right] u_{l}(R)=0
$$

subject to the boundary conditions $u_{l}(0)=0$ and

$$
\begin{aligned}
u_{l}(R) & \sim k R\left[j_{l}(k R) \cos \eta_{l}-n_{l}(k R) \sin \eta_{l}\right] \\
& \sim \sin \left(k R-\frac{l \pi}{2}+\eta_{l}\right) \text { as } R \rightarrow \infty
\end{aligned}
$$

where $j_{l}$ and $n_{l}$ refer to spherical Bessel and Neumann functions, respectively.

[5] The total elastic cross section is given by

$$
\sigma(\varepsilon)=\frac{4 \pi}{k^{2}} \sum_{l=0}^{\infty}(2 l+1) \sin ^{2} \eta_{l} .
$$

The diffusion cross section, which is defined as

$$
\sigma_{d}(\varepsilon)=\frac{4 \pi}{k^{2}} \sum_{l=0}^{\infty}(2 l+1) \sin ^{2}\left(\eta_{l}-\eta_{l+1}\right)
$$

is commonly used for the description of energy transport processes.

[6] In the presence of multiple potentials, statistical cross sections are obtained from the weighted sum of the contributions from each individual molecular channel. The statistical weights for the different electronic states of the OH molecule, ${ }^{2} \Pi,{ }^{2} \Sigma^{-},{ }^{4} \Pi$, and ${ }^{4} \Sigma^{-}$are $2 / 9,1 / 9,4 / 9$, and $2 / 9$, respectively.

[7] Knowledge of the accurate differential cross sections is important for the description of the spatial relaxation of hydrogen or deuterium atoms in oxygen gas. The diffusion coefficient is [Chapman and Cowling, 1939]

$$
D(T)=\frac{3}{16\left(n_{O}+n\right)}\left(\frac{2 \pi k_{B} T}{\mu}\right)^{\frac{1}{2}} \frac{1}{\bar{\sigma}_{d}(T)}
$$

where $T$ is the temperature in Kelvin, $n_{\mathrm{O}}$ is the number density of oxygen atoms, $n$ is the corresponding number density of the hydrogen or deuterium atoms, $k_{\mathrm{B}}$ is Boltzmann's constant, and $\bar{\sigma}_{d}(T)$ is the averaged cross section given by the quadrature over $x=\varepsilon / k_{\mathrm{B}} T$

$$
\bar{\sigma}_{d}(T)=\frac{1}{2} \int_{0}^{\infty} x^{2} \sigma_{a v}(x) e^{-x} d x,
$$

where $\sigma_{\mathrm{av}}$ is the weighted sum of the diffusion cross sections for scattering by the four electronic states associated with $\mathrm{O}\left({ }^{3} P\right)$ and $\mathrm{H}\left({ }^{2} S\right)$.

[8] The energy relaxation of fast atoms due to elastic and inelastic collisions with bath gases has been considered with realistic atomic cross sections by Kharchenko et al. [1997, $1998,2000]$. The rate of energy transitions $\left(E^{\prime} \rightarrow E\right)$ in the laboratory frame (LF) is given by the kernel of the Boltz- mann equation, $B\left(E \mid E^{\prime}\right)$, where $E^{\prime}$ and $E$ are the initial and final energies of the energetic atom. In a uniform bath gas, whose density is much higher than that of the projectile atoms, the energy distribution $f(E, t)$ of the projectile can be described by the linear Boltzmann equation

$$
\begin{aligned}
\frac{\partial}{\partial t} f(E, t)= & \int B\left(E \mid E^{\prime}\right) f\left(E^{\prime}, t\right) d E^{\prime}-f(E, t) \int B\left(E^{\prime} \mid E\right) d E^{\prime} \\
& -\zeta(E) f(E, t)+S(E, t),
\end{aligned}
$$

where $S(E, t)$ is the rate of production of the fast atoms with energy $E$ and $\zeta(E)$ is the rate of the sink reactions that remove them. For binary collisions, elastic or inelastic, $B\left(E \mid E^{\prime}\right)$ has been derived analytically by integrating over all scattering center-of-mass (CM) energies and scattering angles. The rate of collisions is expressed in terms of the quantum mechanically computed double differential cross section $d^{2} \sigma / d \Omega d \varepsilon$ [Kharchenko et al., 1997, 1998]. For elastic collisions, $\varepsilon=\varepsilon^{\prime}$, and $\frac{d \sigma}{d \Omega d \varepsilon}=\frac{d \sigma}{d \Omega} \delta\left(\varepsilon-\varepsilon^{\prime}\right)$. Then

$$
\begin{aligned}
& B\left(E \mid E^{\prime}\right)=\frac{n_{b}}{\left(k_{B} T\right)^{3 / 2}} \sqrt{\frac{2 \pi\left(m+m_{b}\right)}{E^{\prime}}} \frac{\left(m+m_{b}\right)^{2}}{m_{b} m^{2}} \\
& \times \exp \left(\frac{E^{\prime}-E}{2 k_{B} T}-\frac{m_{b}}{m} \frac{E+E^{\prime}}{2 k_{B} T}\right) \\
& \quad \times \int_{0}^{\infty} d \varepsilon e^{-\varepsilon / k_{B} T} \int_{\chi_{\min }}^{\chi_{\max }} \frac{\sin \chi d \chi}{\sqrt{2 \varepsilon-2 \varepsilon \sqrt{\cos \chi}}} \frac{d^{2} \sigma(\varepsilon, \cos \chi)}{d \Omega_{r}} \\
& \quad \times \mathbf{I}_{0}\left(\frac{m_{b}}{m} \frac{\varepsilon}{k_{B} T} \frac{\sin \chi}{1-\cos \chi} \sqrt{\left(\cos \chi-\cos \chi_{+}\right)\left(\cos \chi_{-}-\cos \chi\right)}\right) \\
& \quad \times \exp \left(-\frac{m_{b}}{m} \frac{\varepsilon}{k_{B} T} \cos \chi\right),
\end{aligned}
$$

where the maximal and minimal scattering angles $\chi_{\max }$ and $\chi_{\min }$ are calculated for fixed values of initial and final energies in the laboratory (LF) and center of mass (CMF) frames from the conditions

$$
\chi_{\max }=\min \left\{\pi, \chi_{+}\right\}, \chi_{\min }=\max \left\{-\pi, \chi_{-}\right\}
$$

and $\chi_{ \pm}$are defined by the formal relations

$$
\cos \chi_{ \pm}=1-\frac{m}{2 \mu \varepsilon}\left(\sqrt{E^{\prime}} \pm \sqrt{E}\right)^{2}
$$

For fixed projectile LF energies $E^{\prime}$ and $E$ the critical angles have to satisfy the conditions: $\cos \chi_{-} \geq-1$ and $\cos \chi_{+} \leq+1$, which result in the following restrictions on the $\mathrm{CMF}$ energies $\varepsilon_{\mathrm{r}}$ and $\varepsilon_{\mathrm{r}}^{\prime}$

$$
\sqrt{\varepsilon} \geq \frac{1}{2} \sqrt{\frac{m}{\mu}}\left|\sqrt{E^{\prime}}-\sqrt{E}\right| .
$$


The modified Bessel function $\mathbf{I}_{0}$ in equation (11) results from the analytical integration with respect to the azimuthal angle between the scattering planes in the CMF and LF.

\section{Computational Details}

[9] Four adiabatic electronic terms, ${ }^{2} \Pi,{ }^{2} \Sigma^{-},{ }^{4} \Pi$, and ${ }^{4} \Sigma^{-}$ are involved in the interaction between $\mathrm{O}\left({ }^{3} P\right)$ and $\mathrm{H}\left({ }^{2} S\right)$ [Cooper et al., 1984]. We calculated these potentials using the internally contracted multireference configuration interaction method (MRCISD) [Knowles and Werner, 1988; Werner and Knowles, 1988]. The multireference Davidson's correction $(\mathrm{Q})$ has been applied to account, partially, for the effect of high-order corrections [Davidson and Silver, 1977]. The remaining size consistency errors were approximately eliminated by computing the energies relative to the $\mathrm{H}\left({ }^{2} S\right)+\mathrm{O}\left({ }^{3} P\right)$ asymptote. The MRCISD calculations were based on complete active space self-consistent field (CASSCF) reference wave functions with an active space which consisted of the $\mathrm{H} 1 s$ orbital and the $1 s 2 s 2 p$ orbitals of the $\mathrm{O}$ atom, a total of nine active electrons in six active orbitals. An augmented correlation consistent valence sextuple, zeta basis set (aug-cc-pCV6Z) [Wilson et al., 1996; Woon and Dunning, 1994] was used in MRCISD(Q) calculations. All the calculations were carried out using MOLPRO2006.1 suite of program.

[10] The computed ab initio electronic potential points were fitted by analytical expressions of the Degli-EspostiWerner type [Esposti and Werner, 1990] composed of the short-range term $\left(V_{\mathrm{sh}}\right)$ and the asymptotic long-range part $\left(V_{\mathrm{as}}\right) V(R)=V_{\mathrm{sh}}(R)+V_{\mathrm{as}}(R)$, where

$$
V_{s h}(R)=\sum_{i=0}^{8} a_{i} R^{i} e^{-\alpha(R-\beta)}
$$

and the long-range part was represented by the damped dispersion term

$$
V_{a s}(R)=\frac{1}{2}[1+\tanh (\gamma+\lambda R)] \sum_{n=0}^{4} \frac{C_{2 n+6}}{R^{2 n+6}} .
$$

The nonlinear $\alpha, \beta, \gamma, \lambda$ and the linear $a_{i}$ and $C_{n}$ parameters were optimized using the Levenberg-Marquardt least square algorithm with no constraints imposed. The maximum rootmean square (RMS) error is $8.0 \mathrm{~cm}^{-1}$, which occurs over an energy range around $100 \mathrm{eV}$. The RMS error averaged over the internuclear distance does not exceed $0.3 \mathrm{~cm}^{-1}$. The accuracy of the fitted potentials is high enough for the present study of the energy transfer kinetics of $\mathrm{O}+\mathrm{H}$ collisions in gases at temperatures above $200 \mathrm{~K}$.

[11] The solution of the time-dependent linear kinetic equation (8) may be written

$$
f(E, t)=\int_{0}^{t} \int_{0}^{\infty} K\left(E, t \mid E^{\prime}, t^{\prime}\right) S\left(E^{\prime}, t^{\prime}\right) d E^{\prime} d t^{\prime}
$$

where the propagator $K\left(E, t \mid E^{\prime}, t^{\prime}\right)$ describes the timedependent thermalization of a single atom created at time $t^{\prime}$ with energy $E^{\prime}$. The propagator can be obtained from the solution of equation (8) in which the source function $S(E, t)=\delta\left(E-E^{\prime}\right) \delta\left(t-t^{\prime}\right)$. If the initial distribution function $f(E, t=0)$ is given, $f(E, t)$ at a later time $t$ may be calculated from the propagator $K\left(E, t \mid E^{\prime}, t^{\prime}\right)$ according to

$$
f(E, t)=\int K\left(E, t \mid E^{\prime}, 0\right) f\left(E^{\prime}, 0\right) d E^{\prime} .
$$

The present study is based on the direct numerical solution of the discretized Boltzmann equation. The function $f(E, t)$ is defined in the energy interval $\left[0, E_{\max }\right]$ where $E_{\max }$ is the upper limit to the energy of the nascent atoms. The discretized energy distribution function was represented by the vector $\mathbf{f}$ with the dimensionality $N=E_{\max } / \delta E$ and the kernel $\mathbf{A}$ of the discretized kinetic equation is given by a $N \times N$ matrix [Oppenheim et al., 1977]

$$
A_{i, j}=\delta E\left[B(i \delta E \mid j \delta E)-\delta_{i, j} \omega(i \delta E)-\delta_{i, j} \zeta(i \delta E, T)\right],
$$

where $\delta_{i, j}$ is the Kronecker delta symbol and $\omega(E)=$ $\int_{0}^{\infty} B\left(E^{\prime} \mid E\right) d E^{\prime}$. For any given initial distribution function $\mathbf{f}(0)$ the matrix $\exp \left[\left(t-t^{\prime}\right) \mathbf{A}\right]$ is the discrete representation of the $K$ propagator, and it generates the time-dependent distribution function $\mathbf{f}(t)=\exp [t \mathbf{A}] \mathbf{f}(0)$ [Kharchenko and Dalgarno, 2004]. We obtain the time-dependent average translational energy using the corresponding time-dependent distribution function $f(E, t)$ by

$$
\langle E\rangle=\int f(E, t) E d E .
$$

This time-dependent translational energy is an important parameter in the interpretation of energy relaxation processes.

\section{Results and Discussion \\ 4.1. Interaction Potentials}

[12] The nonlinear least squares fitted parameters for the four corresponding potentials are given in Table 1. The vibrational levels were obtained using the standard DVR procedure [Colbert and Miller, 1992]. The derived dissociation energy for the $\tilde{X}^{2} \Pi$ state of OH is $35290 \mathrm{~cm}^{-1}$, which is consistent with experimental data ranging from 35420 to $35593 \mathrm{~cm}^{-1}$ [Joens, 2001; Ruscic et al., 2002]. The longrange parts of these potentials are governed by the van der Waals interaction. The least squares fitted $C_{6}$ dispersion coefficients differ by no more than $1 \%$ from the values derived from time-dependent linear response density functional theory. Previous $a b$ initio calculations provided only data up to 5.25 Bohr. The shallow attractive parts of the three repulsive potentials were added using the calculated $C_{6}$ dispersion coefficients [Jamieson et al., 2006].

[13] The derived vibrational frequency, $3713 \mathrm{~cm}^{-1}$, agrees closely with the experimental value of $3738 \mathrm{~cm}^{-1}$. The energies of the first 10 computed vibrational levels relative to the $v=0$ level are listed in Table 2 along with available experimental data [Huber and Herzberg, 1979]. The values calculated using Yarkony's [1992] potentials are 
Table 1. Parameters for the Four Interaction Potentials ${ }^{\mathrm{a}}$

\begin{tabular}{lcccc}
\hline & ${ }^{2} \Pi$ & ${ }^{2} \Sigma^{-}$ & ${ }^{4} \Sigma^{-}$ & ${ }^{4} \Pi$ \\
\hline$\alpha$ & 3.9646 & 2.4746 & 3.2605 & 3.2982 \\
$\beta$ & 1.6961 & 1.4473 & 1.7345 & 1.2245 \\
$\gamma$ & -20.7536 & -14.9957 & -13.9861 & -14.2817 \\
$\lambda$ & -4.9957 & -7.2782 & -6.4511 & -7.3934 \\
$a_{0}$ & 0.0826 & 1.2992 & 0.1103 & 0.6772 \\
$a_{1}$ & -0.2045 & -2.8975 & 0.0493 & -0.2228 \\
$a_{2}$ & 0.4345 & 3.6659 & -0.4418 & -1.3736 \\
$a_{3}$ & -0.5433 & -2.7651 & 0.7658 & 3.3054 \\
$a_{4}$ & 0.4148 & 1.2072 & -0.6406 & -3.2161 \\
$a_{5}$ & -0.1895 & -0.2778 & 0.2821 & 1.6278 \\
$a_{6}$ & 0.0317 & 0.0331 & -0.0599 & -0.3770 \\
$a_{7}$ & 0.0050 & -0.0015 & 0.0055 & 0.0376 \\
$a_{8}$ & -0.0033 & $2 \times 10^{-7}$ & $-3 \times 10^{-6}$ & $-5 \times 10^{-7}$ \\
$C_{6}$ & -9.211 & -10.249 & -10.288 & -9.171 \\
$C_{8}$ & 675.78 & -140.31 & -258.76 & -123.28 \\
$C_{10}$ & $-109,329.5$ & 3788.9 & 4491.5 & 2333.8 \\
$C_{12}$ & $3,402,253$ & $-21,742$ & $-21,391$ & $-10,547$ \\
$C_{14}$ & $-29,524,083$ & 38,792 & 32,602 & 15,171 \\
\hline
\end{tabular}

${ }^{\text {a }}$ Parameters are given in atomic units. The current parameters are valid for energies lower than $100 \mathrm{eV}$.

also given in Table 2. Compared to the previous potential the present one agrees better with experimental results. The overall deviations from the experimental levels are smaller than $1 \%$.

[14] The crossings between the ${ }^{2} \Sigma^{-}$and ${ }^{4} \Pi$ states are also found in the current calculation. The two crossing points are 3.74 and 8.33 bohr. In this range, the potential energy of the ${ }^{2} \Sigma^{-}$state lies above that of the ${ }^{4} \Pi$ state, in agreement with the potentials of Jamieson et al. [2006].

\subsection{Cross Sections}

[15] The scattering problem was solved using the standard renormalized Numerov method and the phase shift was determined from the asymptotic expression of the Bessel and Neumann functions of equation (3). The convergence was carefully checked with respect to the integration step length and the number of partial waves. Total cross sections for each individual electronic state were then determined through equation (4) and the optical theorem was used to further check the accuracy of the calculation. Summing up the cross sections of those four individual potentials with their corresponding statistical weights then yielded the overall effective cross sections.

[16] The calculated total cross sections for the four potentials are shown in Figure 1 along with the results from the previous calculation using Yarkony's [1992] potentials. The present results are systematically smaller than the earlier ones by about $10 \%$. The largest deviation occurs for the $\tilde{X}^{2} \Pi$ state. The deeper potential wells in Yarkony's [1992] potentials cause this difference. The structures of the glory oscillations for the $\tilde{X}^{2} \Pi$ state in the scattering distributions are not very different except that their positions are shifted, which is understandable because of the similarity in these two sets of potentials. Overall, these two results agree reasonably well with each other, which validates both calculations. The new result is presumably more accurate thanks to the improved methodologies employed in the calculations of the potential energy curves.

[17] The calculated effective differential cross sections (DCSs) at selected scattering energies ranging from 0.1 to $11.0 \mathrm{eV}$ are presented in Figure 2. The fast oscillations with scattering angles reflect quantum mechanical interferences. There are substantial contributions from large-scattering angles at low energies, but they decrease quickly with the increase of scattering energy. The oscillation also decreases quickly with the increase of scattering angle. The behavior of the oscillations at relatively high energy can be described by the quasi-classical scattering theory, as illustrated in Figure 3, where we present DCSs near the classical rainbow angle from both quasi-classical and quantum mechanical calculations at a scattering energy $\varepsilon=9.0 \mathrm{eV}$ in the $\tilde{X}^{2} \Pi$ ground state. The rainbow angle $\chi_{r}$ corresponds to the maximum or minimum of the deflection angle which can be determined from the quasi-classical expression of a scattering angle via the partial wave phase shift $\eta_{l}$

$$
\chi=2 \frac{d \eta_{l}}{d l}, \text { where }\left.\frac{d \chi_{r}}{d l}\right|_{l=l_{r}}=0
$$

The quasi-classical DCS can then be expressed as [Landau and Lifshitz, 1991]

$$
\frac{d \sigma}{d \Omega}=\frac{2 l_{r}}{k^{2} \alpha^{2 / 3} \sin \chi} A i^{2}\left(\frac{\chi-\chi_{r}}{\alpha^{2 / 3}}\right)
$$

where $\alpha=\left.\frac{d^{3} \eta_{l}}{d l^{3}}\right|_{l=l_{r}}$ and $A i$ is the Airy function. The close agreement between quasi-classical and quantum mechanical results, averaged over the fast oscillations near the rainbow angle indicate that the fast decay of the oscillation with the increase of scattering energy may be represented by the Airy function. The amplitude of the Airy function decreases very fast in the classically forbidden region $\left(\chi>\chi_{r}\right)$. There are also high-frequency (fast) and small-amplitude oscillations in the QM calculation. These oscillations are the result of the interference between scattering at small and large impact parameters which yield the same scattering angle with different phases.

[18] In Figure 4, we present a three-dimensional plot of the DCS as functions of scattering angle and energy, which illustrates a global characteristic of oscillations of the differential cross sections in energy and scattering angle. It provides necessary information for the evaluation of momentum-energy transfer in collisions of $\mathrm{O}$ and $\mathrm{H}$ gases.

[19] Isotope fractionations in atmospheric escape is an important issue for the evolution of planetary atmospheres. The isotope dependence of the DCS was examined and the

Table 2. Energies and Energy Differences Between Theory and Experiment for the First 10 Vibrational Levels of the $\tilde{X}^{2} \Pi$ State of $\mathrm{O}^{16} \mathrm{H}^{1 \mathrm{a}}$

\begin{tabular}{cccc}
\hline Level & Experiment $(\mathrm{eV})$ & Yarkony's [1992] Potentials $^{\mathrm{b}}$ & This Work $^{\mathrm{b}}$ \\
\hline 1 & 0.44409 & -0.00136 & 0.00149 \\
2 & 0.86777 & -0.00280 & 0.00303 \\
3 & 1.27127 & -0.00440 & 0.00447 \\
4 & 1.65466 & -0.00629 & 0.00556 \\
5 & 2.01783 & -0.00882 & 0.00593 \\
6 & 2.36046 & -0.01229 & 0.00516 \\
7 & 2.68205 & -0.01750 & 0.00245 \\
8 & 2.98188 & -0.02494 & -0.00282 \\
9 & 3.25892 & -0.03532 & -0.01138 \\
10 & 3.51180 & -0.05008 & -0.02460 \\
\hline
\end{tabular}

${ }^{\mathrm{a}}$ First 10 vibrational levels are relative to $v=0$ level.

${ }^{\mathrm{b}}$ Numbers in these columns are differences in $\mathrm{eV}$ from experimental results. 

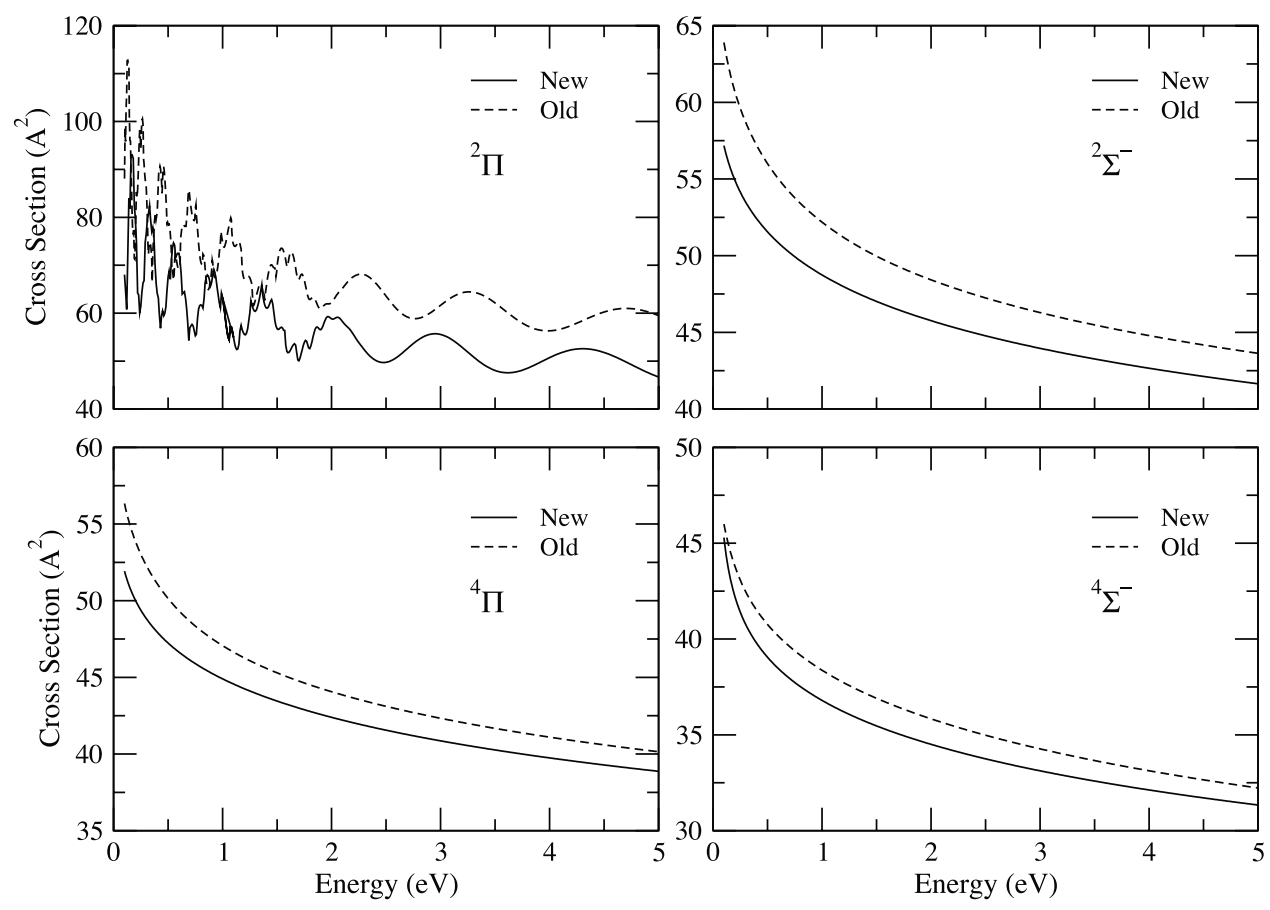

Figure 1. Total elastic cross sections for each of the four electronic states of $\mathrm{OH}$. The solid lines are the results from the potentials reported in the present work, and the dashed lines are calculated using Yarkony's [1992] potentials.

results are given in Figure 5 for two scattering energies of 1.0 and $10.0 \mathrm{eV}$. No significant difference was observed. The major differences between $\mathrm{O}+\mathrm{H}$ and $\mathrm{O}+\mathrm{D}$ are small shifts in the positions of oscillations, which reflect the difference in the vibrational structure of the two molecular species, $\mathrm{OH}$ and OD.
[20] The total cross sections (TCSs) for collisions of $\mathrm{O}$ with $\mathrm{H}$ and $\mathrm{D}$ as functions of scattering energy are given in Figure 6 and the small differences in the diffusion cross sections between $\mathrm{OH}$ and $\mathrm{OD}$ as a function of scattering energy are shown in Figure 7. As for the DCS, these two TCSs have a similar energy dependence, and the TCS of $\mathrm{OD}$ is about $7 \%$ larger than that of $\mathrm{OH}$. At the same

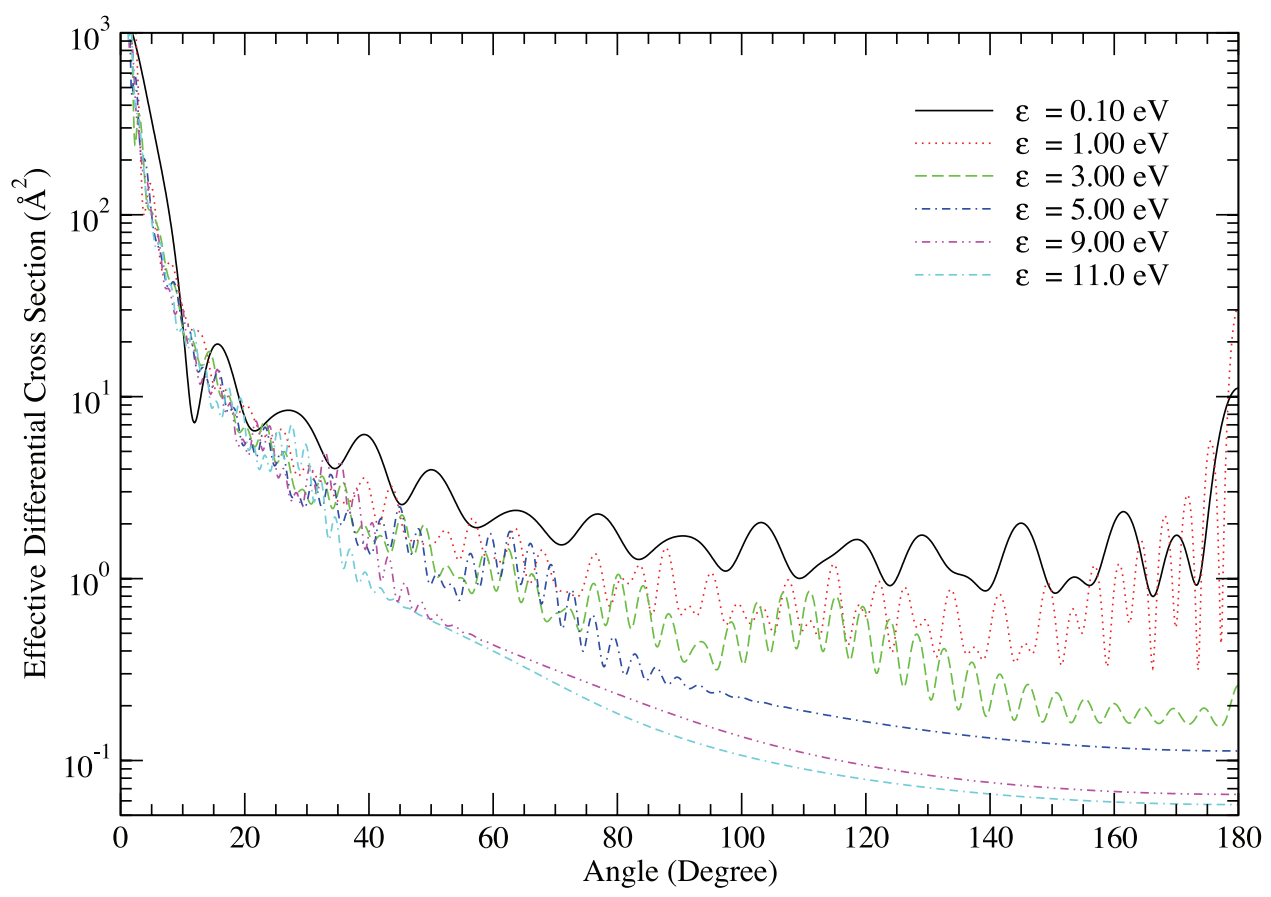

Figure 2. Statistical-weighted differential cross sections at selected energies for $\mathrm{O}-\mathrm{H}$ collisions. 


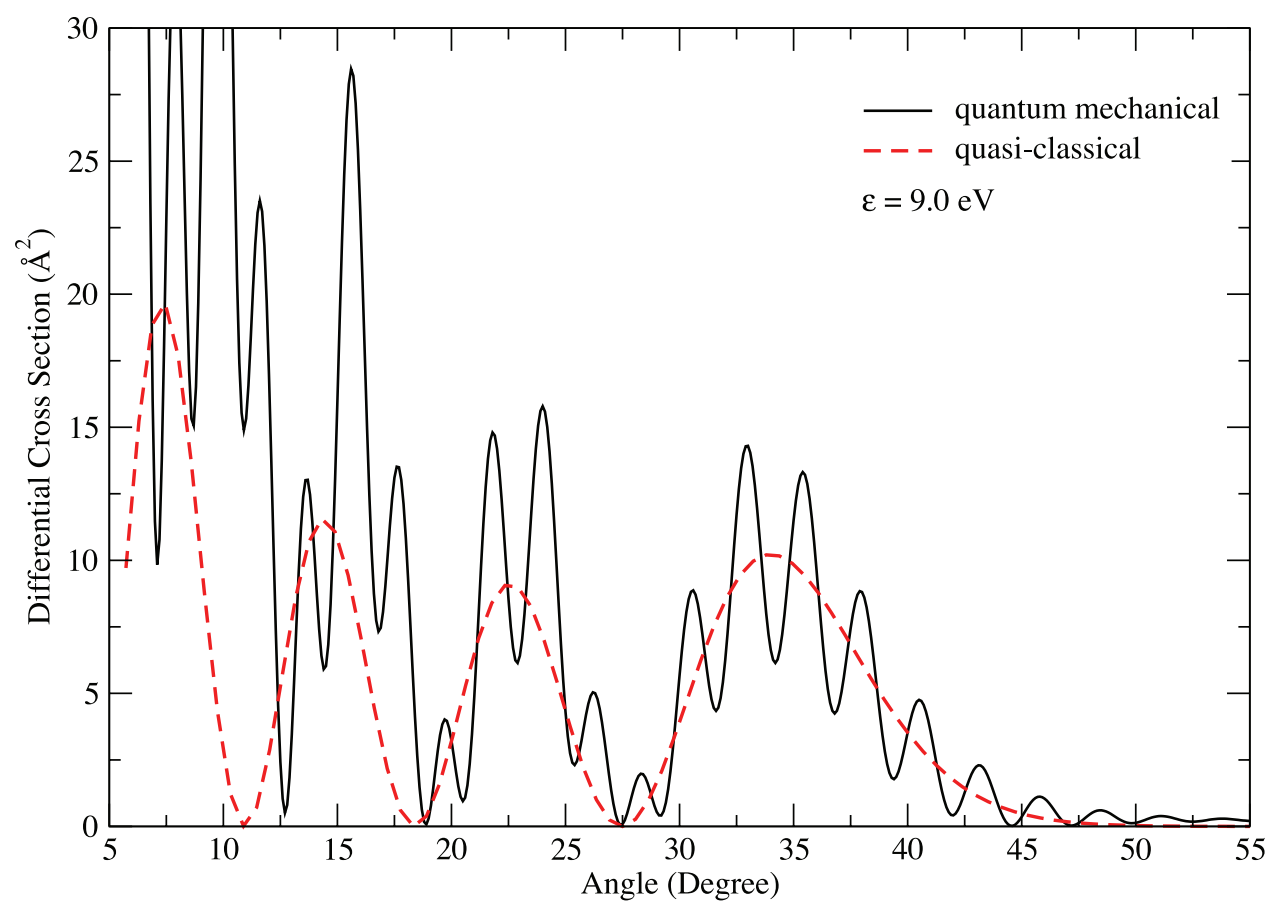

Figure 3. Differential cross sections for the $X^{2} \Pi$ state of the $\mathrm{O}-\mathrm{H}$ collisions at a center of massscattering energy of $9 \mathrm{eV}$. The solid line is calculated fully quantum mechanically, and the dashed line is computed using quasi-classical theory.

collision energy, the larger TCS in OD is due to its heavier reduced mass. In the relative collision velocity representation, the TCSs for both isotopes are comparable as seen in the embedded picture in Figure 6. The diffusion cross section for $\mathrm{OH}$ as a function of scattering energy is shown in Figure 6. Substantial differences with OD exist only in the low-energy regime as shown in Figure 7. Differences in the diffusion cross sections of $\mathrm{OH}$ and $\mathrm{OD}$ are negligible at collision energies above $2 \mathrm{eV}$.

\subsection{Collisional Relaxation}

[21] With quantum mechanically calculated DCSs, we construct the kernel, equation (9), of the Boltzmann equation at different temperatures, bath gas densities, and projectile atoms. Combinations of the projectile and bath gases are consistent with conditions in the Mars and Earth atmospheres. To demonstrate the physical nature of the energy transfer process under different conditions, we present our calculated energy transfer rate for unit bath gas density $\left(1 \mathrm{~cm}^{-3}\right)$, unless otherwise specified. Actual kernels for atmospheric conditions maybe obtained by a simple multiplication of the present results with the actual atmospheric gas density.

[22] The kernels of the Boltzmann equation for energetic $\mathrm{O}$ moving in $\mathrm{H}$ or $\mathrm{D}$ bath gases calculated at selected initial LF energies as a function of final LF energies at different temperatures are presented in Figure 8. The sharp maxima occurring at $E \sim E^{\prime}$ reflect an elastic cross section dominated by small-angle scattering involving small energy loss. They cannot be described by the hard-sphere approximation [Kharchenko et al., 1997, 1998; Zhang et al., 2007]. To illustrate the dependence of the thermalization kinetics on the temperature and the mass ratio between the projectile and the bath gas atoms, the frequencies $\omega(E)$ of energy transfer collisions and the temperature-dependent energy loss rate $\gamma(E, T)$ obtained through the Boltzmann kernel $B\left(E \mid E^{\prime}\right)$ by

$$
\omega(E)=\int B\left(E^{\prime} \mid E\right) d E^{\prime}
$$

and

$$
\gamma(E, T)=-\frac{d E}{d t}=\int B\left(E^{\prime} \mid E\right)\left(E-E^{\prime}\right) d E^{\prime}
$$

are shown in Figures 9 and 10, respectively, as functions of $E$. These results can be extended to the bath gas with arbitrary number density $n$ by multiplying $\omega(E)$ and $\gamma(E, T)$ with $n$.

[23] There is a shallow well in the collision frequency for energetic $\mathrm{O}$ traversing the $\mathrm{H}$ and $\mathrm{D}$ bath gases that is the result of a cross section increasing with decreasing collision energy. The temperature dependence of the $\mathrm{O}-\mathrm{H}$ collision energy transfer is weak and the difference in the energy loss rates for hot $\mathrm{O}$ traversing the $\mathrm{H}$ bath gas at $200 \mathrm{~K}$ and 1000 $\mathrm{K}$ is negligible.

[24] If we replace the bath gas $\mathrm{H}$ by $\mathrm{D}$, there is little change in the collision frequency, but a relatively large effect on the energy loss rate, which occurs because the most efficient energy transfer takes place when the projectile and bath gas atoms have similar masses. We define the mass ratio $r_{m}=m_{p} / m_{b}$, where $m_{b}$ and $m_{p}$ are the masses of the bath gas atoms and the projectile atom, respectively. The mass ratio $r_{m}$ in the bath gas $\mathrm{D}$ is closer to unity than in the bath gas $\mathrm{H}$. Thus, the more efficient energy transfer collision leads to a large collision energy loss rate. In addition, 


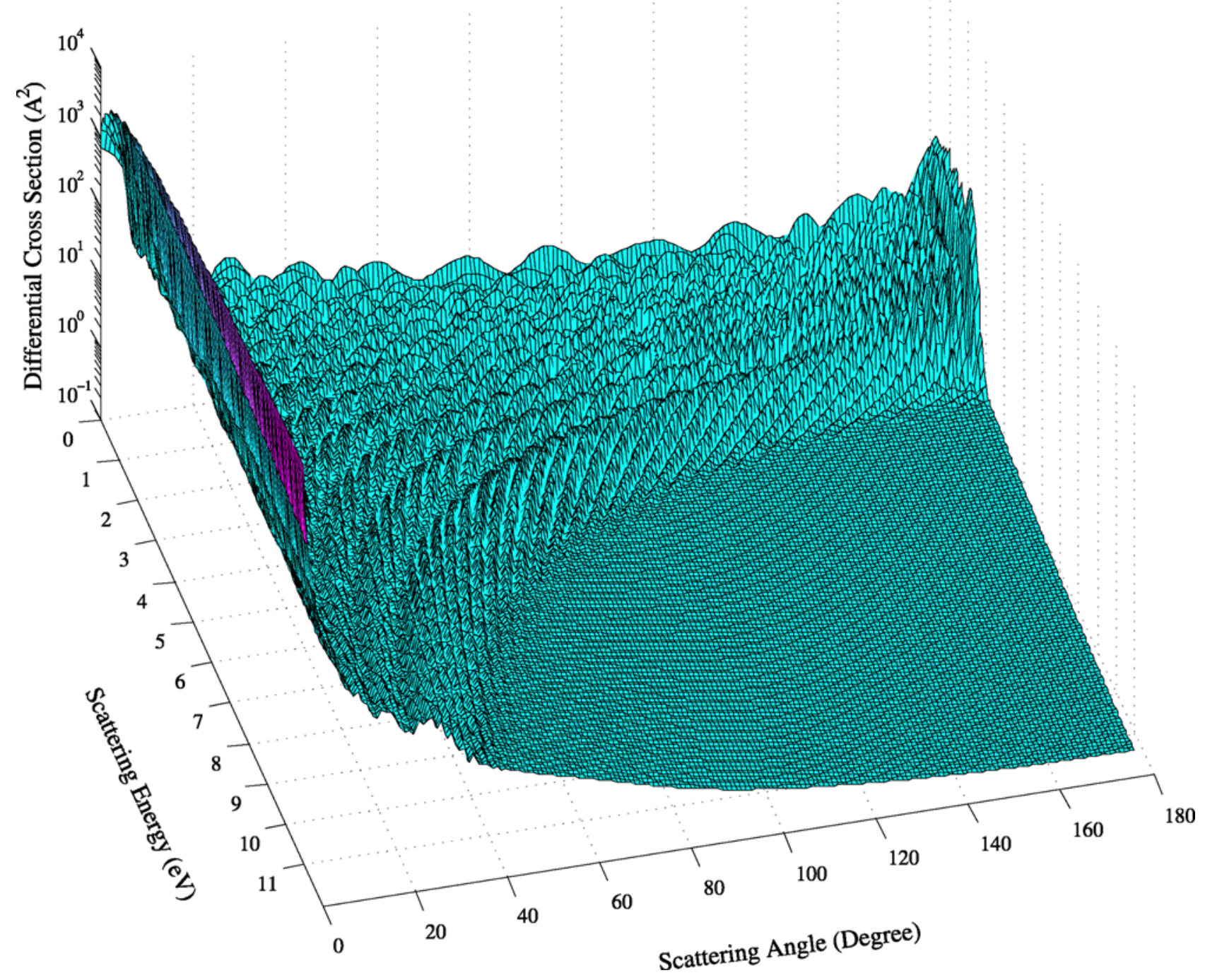

Figure 4. Three-dimensional plot of the statistical-weighted differential cross sections of the O-H collision as functions of center of mass-scattering energies and scattering angles.

the higher the initial energy, the larger the energy loss rate, because of the higher collision frequency in bath gas $\mathrm{D}$ at higher energies. However, the change from $\mathrm{H}$ to $\mathrm{D}$ is small; the energy loss rate difference at the highest energy of $10 \mathrm{eV}$ in the present study does not exceed a factor of two.

[25] Oxygen and hydrogen atoms are present in planetary atmospheres at different altitudes, and nascent energetic $\mathrm{O}$ and $\mathrm{H}$ atoms are thermalized in the equilibrium $\mathrm{O}$ or $\mathrm{H}$ gases. The interchange of projectile and bath gas species modifies the mass ratio $r_{m}$ and therefore influences the energy transfer kinetics. When $r_{m}<1$, as in the case of hot $\mathrm{H}$ or $\mathrm{D}$ atoms moving in the $\mathrm{O}$ bath gas, $\omega(E)$ is higher than in the opposite situation with $r_{m}>1$; therefore the energy loss rate is larger for the light projectile with low initial energies, as demonstrated in Figure 10 with initial energy smaller than $5.8 \mathrm{eV}$ and a bath gas temperature of $1000 \mathrm{~K}$. The ratios of collision frequency changes are roughly the same with further increase of the initial energy. The efficient energy transfer in the collision from heavy to light particles gradually increases the energy loss rate and eventually exceeds the rate for the case with $r_{m}<1$. The crossing point depends on the bath gas temperature and density, the mass ratio $r_{m}$ and the collision cross sections. In the $\mathrm{O}-\mathrm{H}$ system, the temperature has little effect. The crossing point is located approximately at $5.8 \mathrm{eV}$. The energy loss rate difference, however, is small in the current energy range. It is modified for actual conditions by variation of the bath gas density.

[26] The number and energy distributions of the secondary hot atoms is a critical quantity in understanding the atom escape mechanism from planets. The escape energy of ${ }^{16} \mathrm{O}$ is $1.98 \mathrm{eV}$ in the Martian atmosphere and $0.66 \mathrm{eV}$ for hydrogen in the Earth atmosphere. If the recoil atoms gain enough energy, the upward flux of the recoil atoms will contribute to the atom escape. The escape fraction can be determined from the Boltzmann kernel. We are interested in recoil collisions where the energies of the secondary hot atoms $E_{r}$ are significantly larger than the thermal energy. 

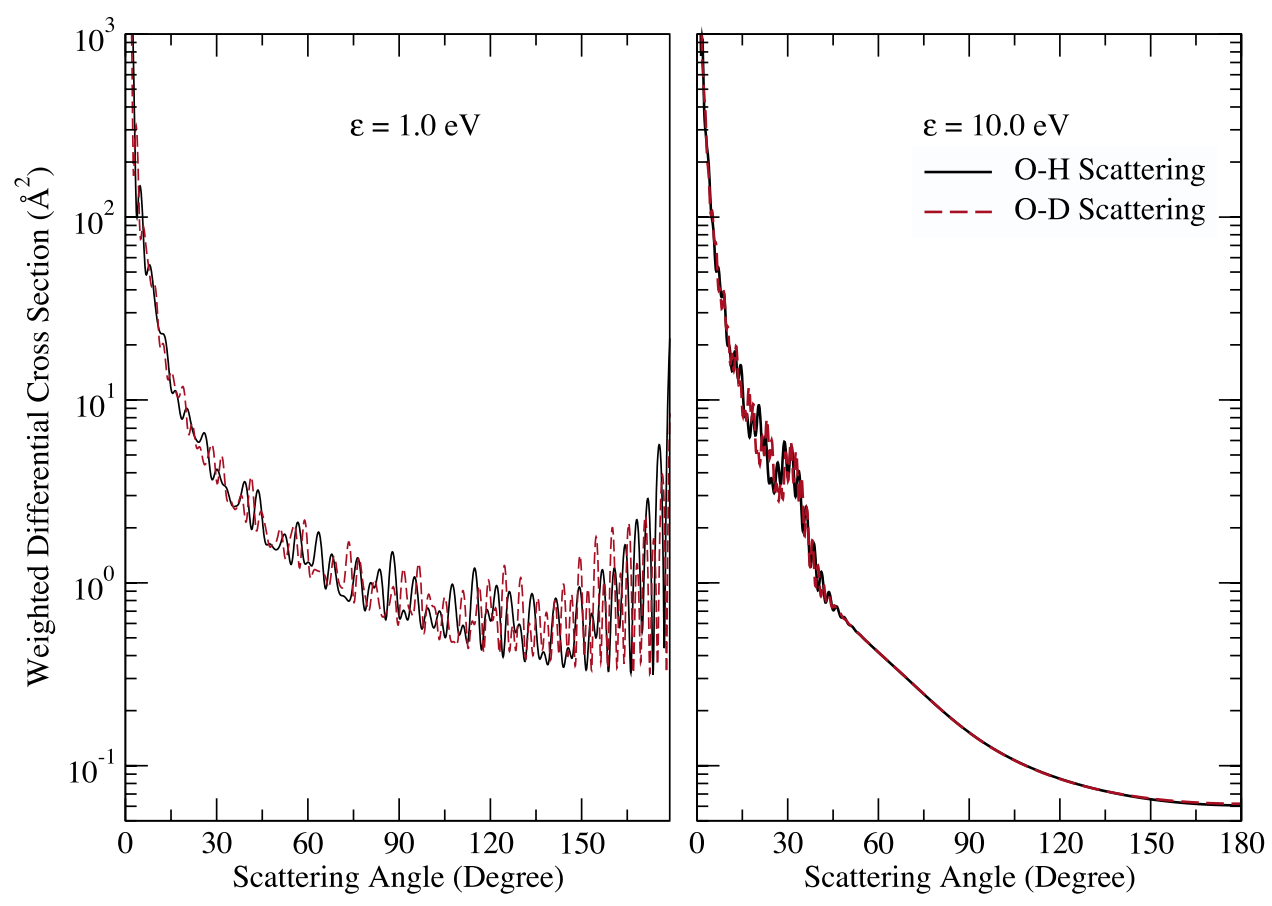

Figure 5. Comparisons of the statistical-weighted differential cross sections of O-H and O-D collisions at center of mass-scattering energies of 1 and $10 \mathrm{eV}$.

Hence the initial energies of the bath gas atoms may be neglected. Normalized to unity the energy distribution function $\rho\left(E_{r} \mid E\right)$ of recoil atoms is given by

$$
\rho\left(E_{r} \mid E\right)=\frac{1}{\omega(E)} B\left(E-E_{r} \mid E\right), \text { with } \int d E_{r} \rho\left(E_{r}, E\right)=1
$$

The calculated energy distributions of recoil atoms at selected initial projectile energies, temperatures, and bath gases are shown in Figure 11. The fraction of secondary hot atoms increases with the initial projectile energy. The fraction of recoil ${ }^{16} \mathrm{O}$ atoms with energy greater than $2 \mathrm{eV}$ at the bath gas temperature $1000 \mathrm{~K}$ changes from nearly zero to $0.9 \%$ as the initial energy of $\mathrm{H}$ atoms increases from 3 to

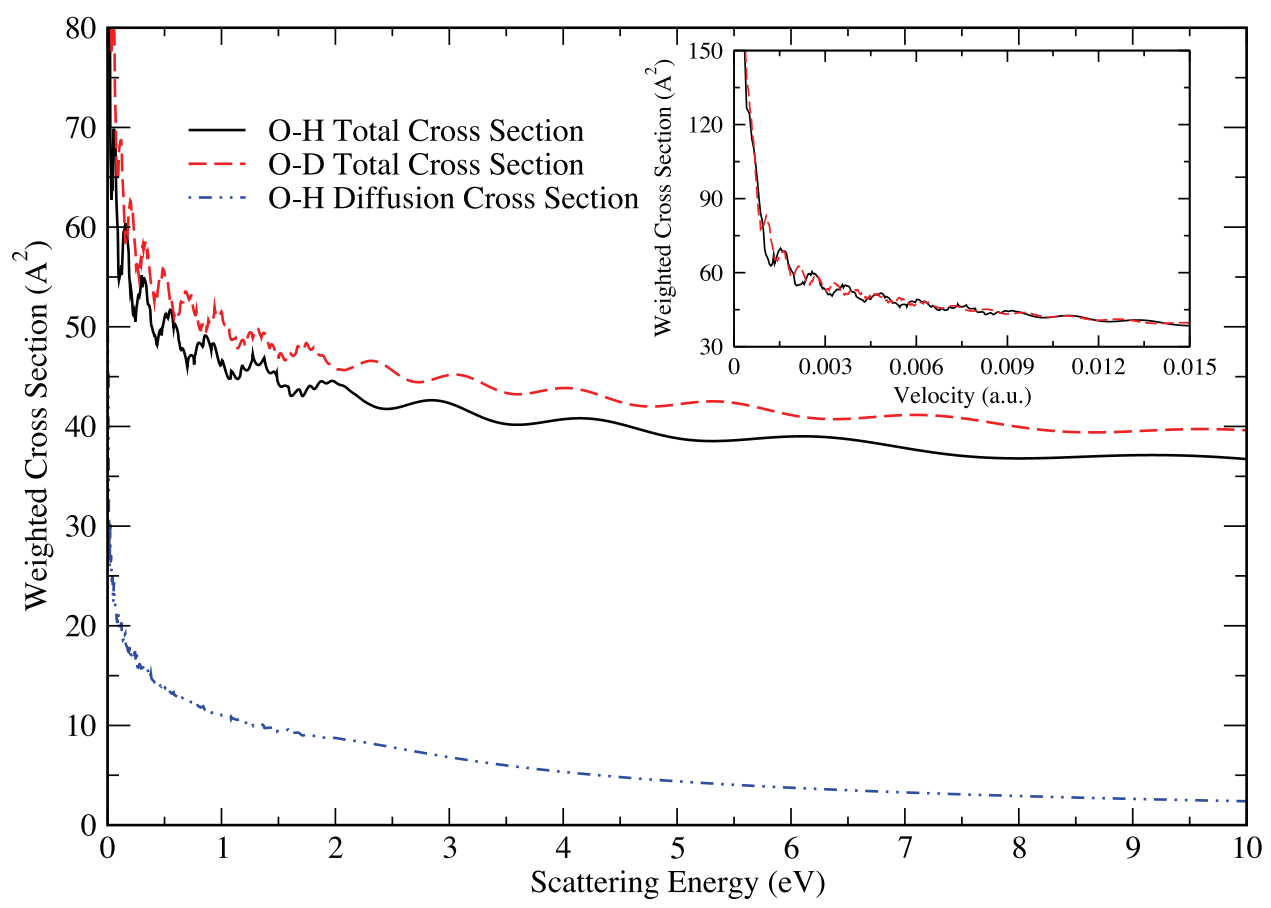

Figure 6. Total cross sections and diffusion cross sections of O-H and O-D collisions as functions of center of mass-scattering energies. 


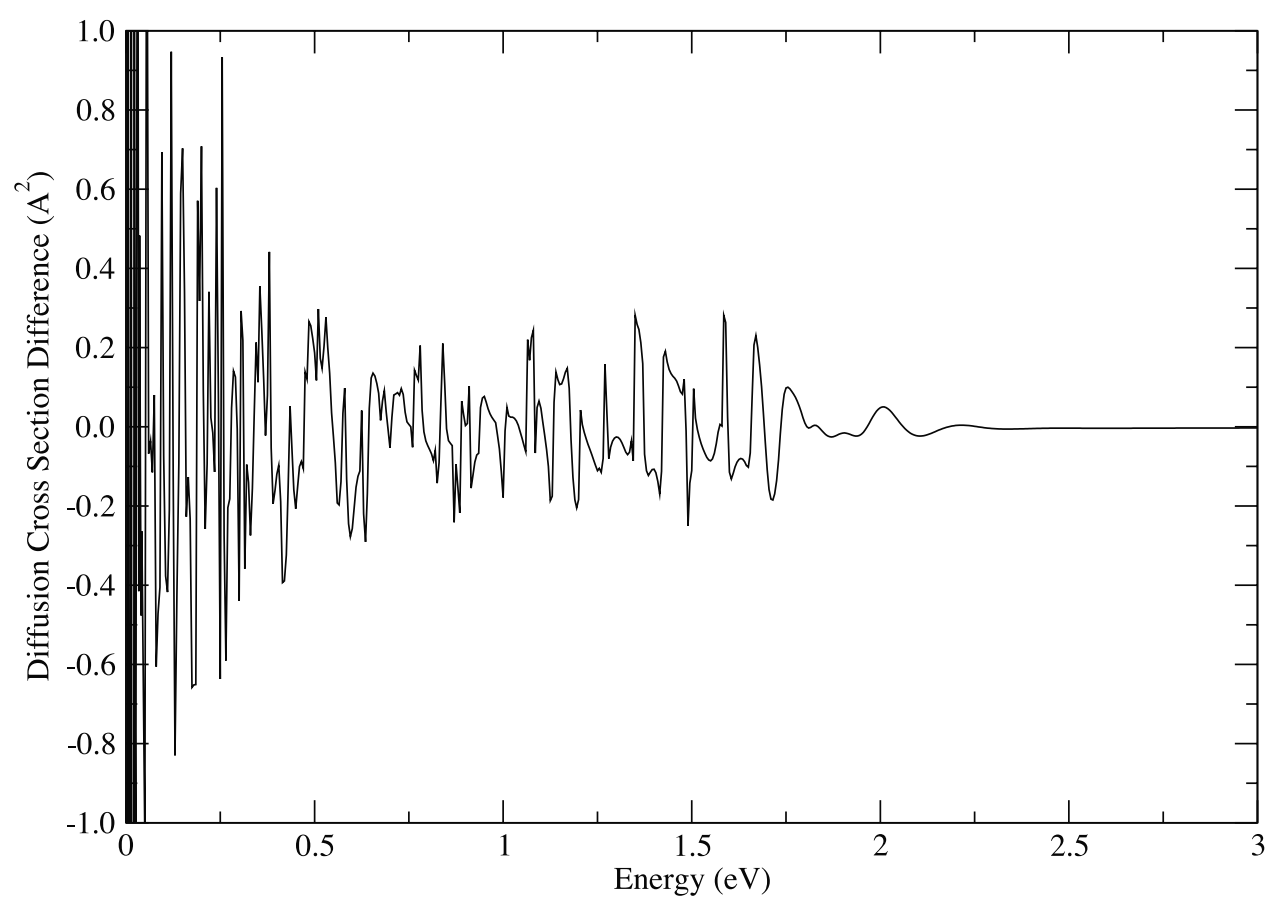

Figure 7. The difference in the diffusion cross sections in O-H and O-D collisions as a function of center of mass-scattering energy.

$9 \mathrm{eV}$. Lowering the bath gas temperature will decrease the fraction of high-energy recoil atoms. Only $0.3 \%$ recoil ${ }^{16} \mathrm{O}$ atoms with energy above $2 \mathrm{eV}$ are created by the hot $\mathrm{H}$ atom with $9.0 \mathrm{eV}$ initial energy.
[27] The $\mathrm{H}$ and $\mathrm{D}$ escape energies on Mars are 0.13 and $0.26 \mathrm{eV}$, respectively. The calculated percentages of the recoil $\mathrm{H}$ and $\mathrm{D}$ atoms, generated by hot $\mathrm{O}$ atoms, with energies above the escape thresholds are listed in Table 3 at

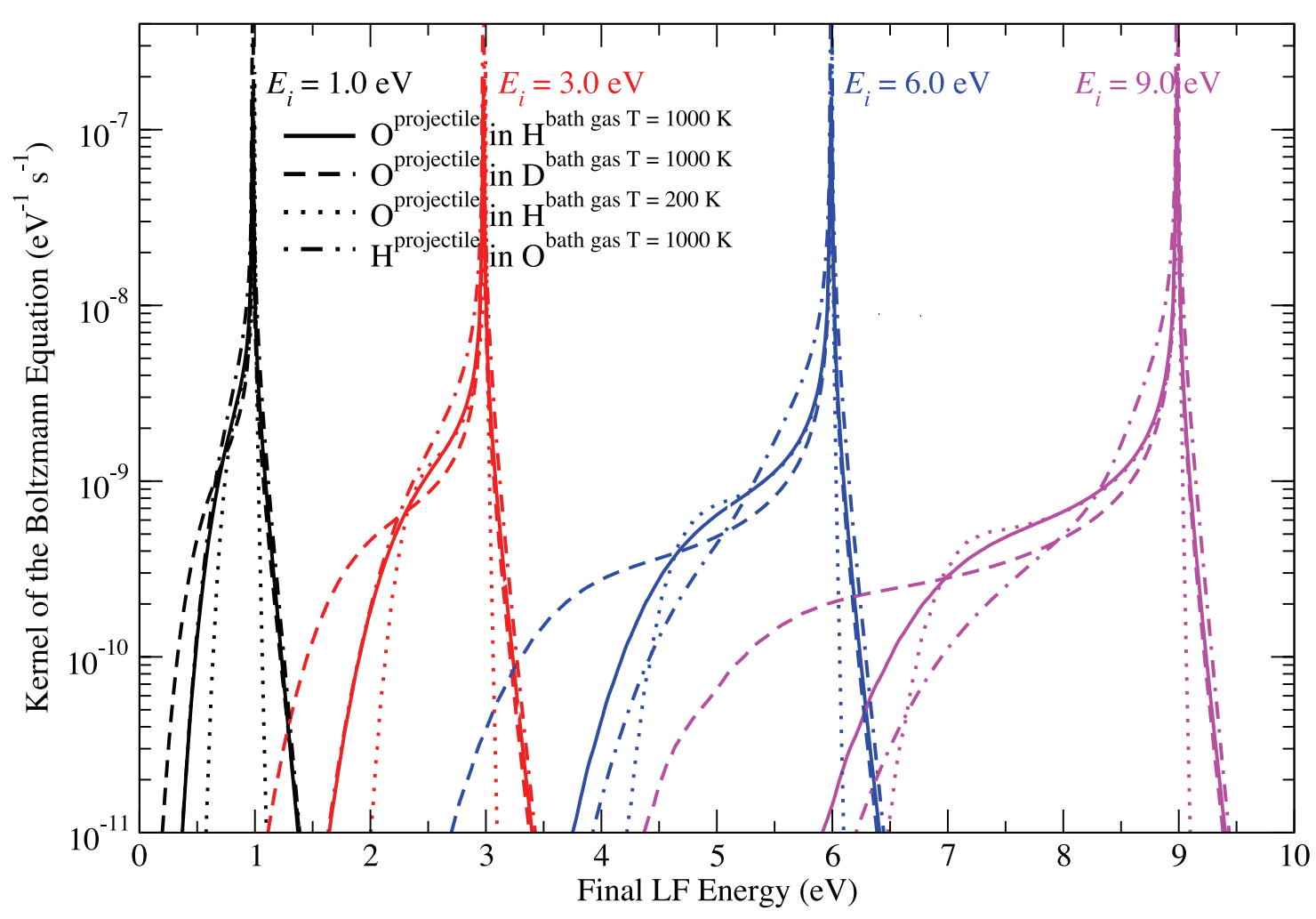

Figure 8. Collision kernels at initial $\mathrm{O}$ or $\mathrm{H}$ laboratory frame energies as functions of final laboratory frame energy at selected temperatures and bath gases. The bath gas density was taken to be $1 \mathrm{~cm}^{-3}$. 


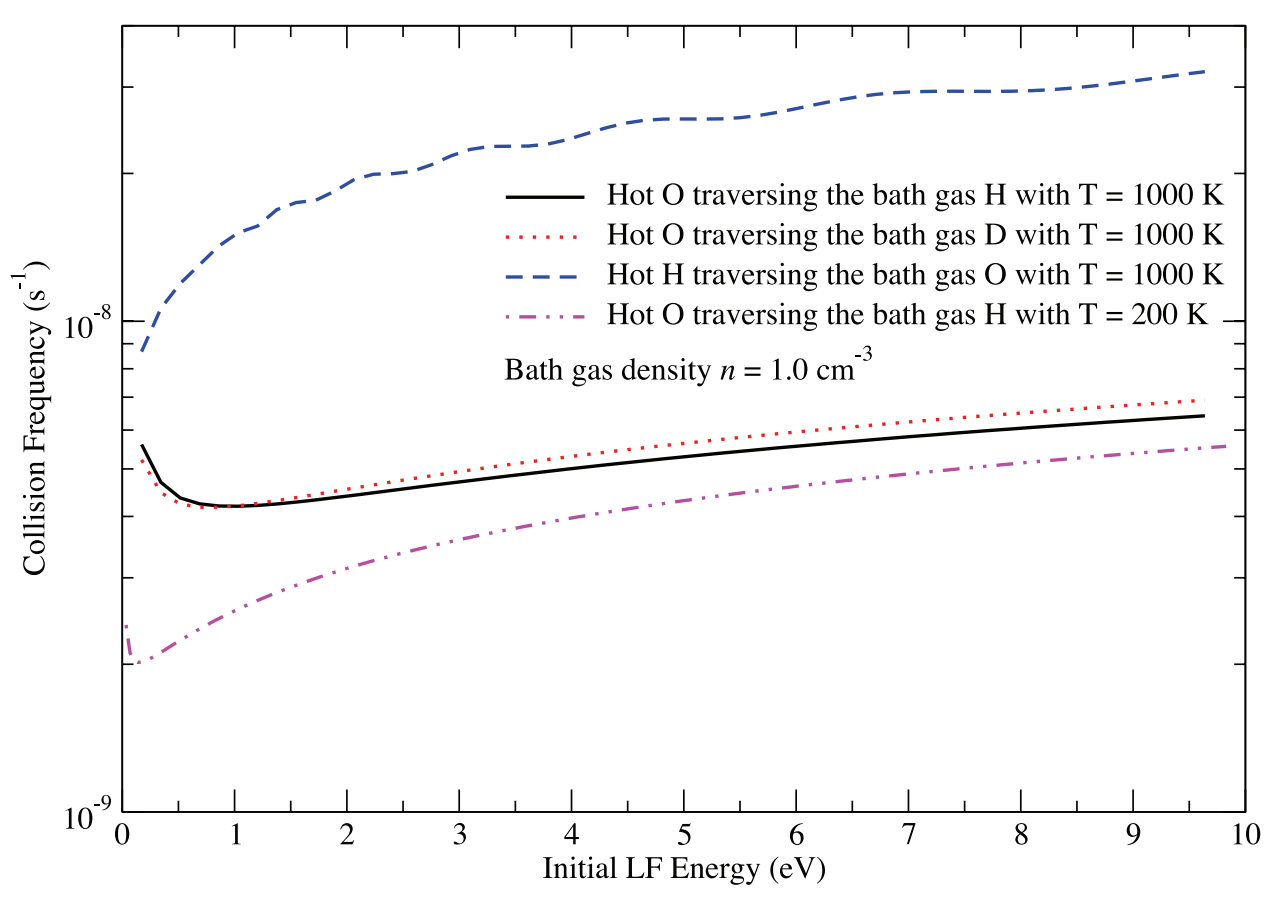

Figure 9. Collision frequencies of fast $\mathrm{O}$ or $\mathrm{H}$ thermalized in $\mathrm{H}, \mathrm{D}$, or $\mathrm{O}$ bath gases at selected bath gas temperatures as a function of initial laboratory frame energy for a bath gas density of unity.

selected bath gas temperatures and initial $\mathrm{O}$ energies. The bath gas temperature of $200 \mathrm{~K}$ occurs on Mars at an altitude of $400 \mathrm{~km}$. Compared to $\mathrm{D}$ atoms, more $\mathrm{H}$ atoms are capable of escape in the same conditions.

[28] The evolution of the energy distributions function of hot atoms may provide an opportunity for direct laboratory measurements of the energy transfer kinetics. The time- dependent averaged translational energy derived from the energy distribution function also serves as a benchmark for yielding effective hard-sphere cross sections to be used in large-scale simulations. As an illustrative example, we studied the thermalization process of energetic $\mathrm{O}$ atoms moving in an $\mathrm{H}$ thermal bath with a temperature of $200 \mathrm{~K}$ and a density of $2.0 \times 10^{5} \mathrm{~cm}^{-3}$, corresponding to atmo-

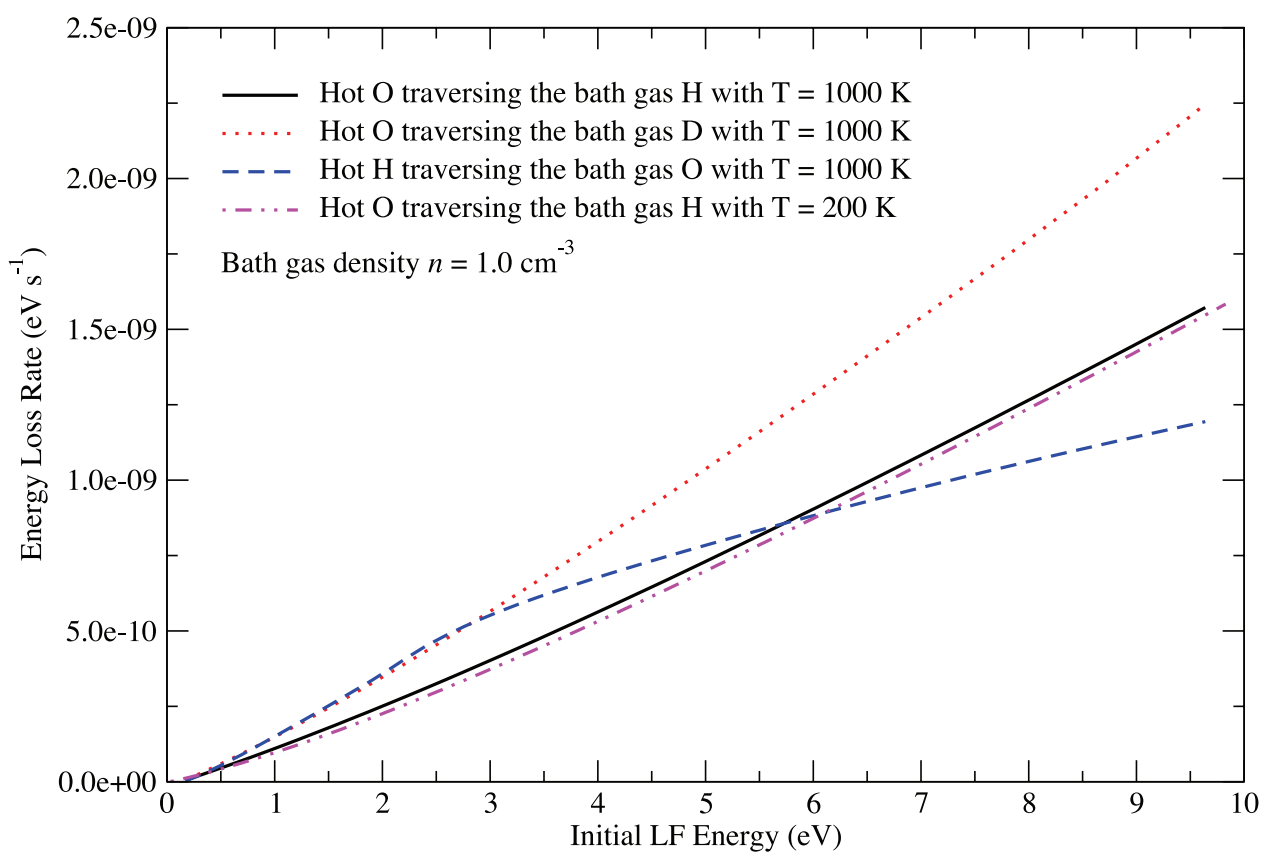

Figure 10. Energy loss rate of fast $\mathrm{O}$ or $\mathrm{H}$ thermalized in $\mathrm{H}, \mathrm{D}$, or $\mathrm{O}$ bath gases at selected bath gas temperatures as a function of initial laboratory frame energy. Unit bath gas density was used in the calculation. 


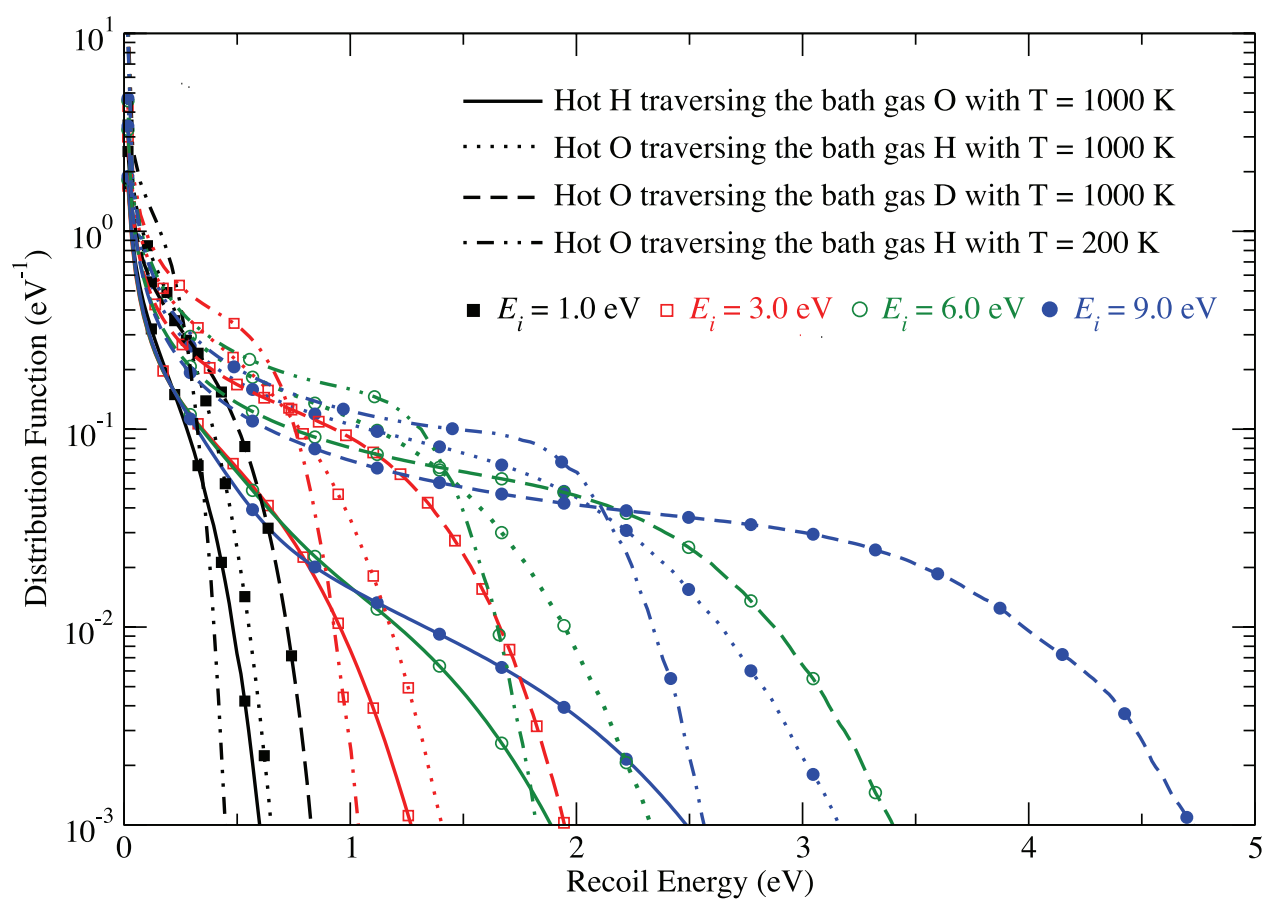

Figure 11. Distribution functions of secondary fast atoms as functions of recoil energy for fast $\mathrm{O}$ or $\mathrm{H}$ thermalized in $\mathrm{H}, \mathrm{D}$, or $\mathrm{O}$ bath gases at selected bath gas temperatures and initial laboratory frame energies at a bath gas density of $1 \mathrm{~cm}^{-3}$.

spheric conditions at an attitude of $400 \mathrm{~km}$ on Mars. Hot $\mathrm{O}$ is produced by the dissociative recombination (DR) of $\mathrm{O}_{2}^{+}$ ions. There are other sources, which we neglect for simplicity. Three channels are associated with the production of energetic $\mathrm{O}\left({ }^{3} P\right)$ in the $\mathrm{DR}$ of $\mathrm{O}_{2}^{+}$[Kharchenko and Dalgarno, 2004; Guberman, 1988]. The exothermicities and branching ratios of different DR channels are listed in the following:

$$
\begin{gathered}
\mathrm{O}_{2}^{+}+e \longrightarrow \mathrm{O}\left({ }^{3} P\right)+\mathrm{O}\left({ }^{3} P\right) 6.65 \mathrm{eV} 0.22 \\
\quad \longrightarrow \mathrm{O}\left({ }^{1} D\right)+\mathrm{O}\left({ }^{3} P\right) 4.99 \mathrm{eV} 0.42 \\
\quad \longrightarrow \mathrm{O}\left({ }^{1} S\right)+\mathrm{O}\left({ }^{3} P\right) 2.77 \mathrm{eV}<0.01
\end{gathered}
$$

These values depend on the vibrational state of $\mathrm{O}_{2}^{+}$and on the electron collision energy. In the current calculation we assume that $\mathrm{O}_{2}^{+}$is in the ground vibrational state and electron collision energies are below $1 \mathrm{meV}$. The resulting initial LF average translational energy of hot $\mathrm{O}$ atoms is $5.57 \mathrm{eV}$. The initial energy distribution function was constructed using the standard formula for the transformation of the monoenergetic distributions from CM to LF and averaged with the corresponding branching ratio [Forrey et al., 1996].

[29] The distribution functions at selected times are shown in Figure 12. The initial double peaks centered around $5 \mathrm{eV}$ and $6.6 \mathrm{eV}$ reflect two major channels in the DR of $\mathrm{O}_{2}^{+}$. The distribution function quickly acquired a Maxwellian-like shape with an averaged kinetic energy much higher than the thermal energy of the bath gas. The rest of the relaxation followed a continuum of Maxwellianlike distribution functions characterized by a time-dependent effective temperature $T(t)$ [Zhang et al., 2008].

[30] We calculated time-dependent average kinetic energies for energetic atoms thermalized in bath gases corresponding to different atmospheric conditions on Earth and Mars. The effective hard-sphere cross sections fitted to reproduce the relaxation kinetics are reported in Table 4. No single hard-sphere cross section can correctly describe the relaxation process, especially, for light particles penetrating into the heavy bath gas, as illustrated in Figure 13 for the D atoms moving in the $\mathrm{O}$ bath gas with $T=1000 \mathrm{~K}$. Therefore, for such cases we fitted the hard-sphere cross sections using step functions by dividing the relaxation process into two or more energy intervals.

[31] A temperature dependence of the fitted hard-sphere cross sections was found in the calculations. As an example we present in Figure 14 the translational energy of hot $\mathrm{H}$ atoms as a function of time in the $\mathrm{O}$ bath gases at three different temperatures, 200,400 , and $1000 \mathrm{~K}$. The energies of the $\mathrm{H}$ atoms are given in units of $k_{\mathrm{B}} T$. The absolute time

Table 3. Percentage of the Secondary Energetic $\mathrm{H}$ and D Atoms With Translation Energies Above $0.13 \mathrm{eV}$ and $0.26 \mathrm{eV}$ Produced by the Initial Hot $\mathrm{O}$ Atom With Different Energies From $1.0 \mathrm{eV}$ to $9.0 \mathrm{eV}$

\begin{tabular}{cccccc}
\hline & \multicolumn{2}{c}{$\mathrm{H}$} & & \multicolumn{2}{c}{$\mathrm{D}$} \\
\cline { 2 - 3 } \cline { 5 - 6 } Energy $(\mathrm{eV})$ & $200 \mathrm{~K}$ & $1000 \mathrm{~K}$ & & $200 \mathrm{~K}$ & $1000 \mathrm{~K}$ \\
\hline 1.0 & $21 \%$ & $35 \%$ & & $16 \%$ & $25 \%$ \\
2.0 & $36 \%$ & $47 \%$ & & $31 \%$ & $40 \%$ \\
3.0 & $42 \%$ & $53 \%$ & & $37 \%$ & $47 \%$ \\
6.0 & $49 \%$ & $61 \%$ & & $42 \%$ & $54 \%$ \\
9.0 & $51 \%$ & $63 \%$ & & $44 \%$ & $56 \%$ \\
\hline
\end{tabular}




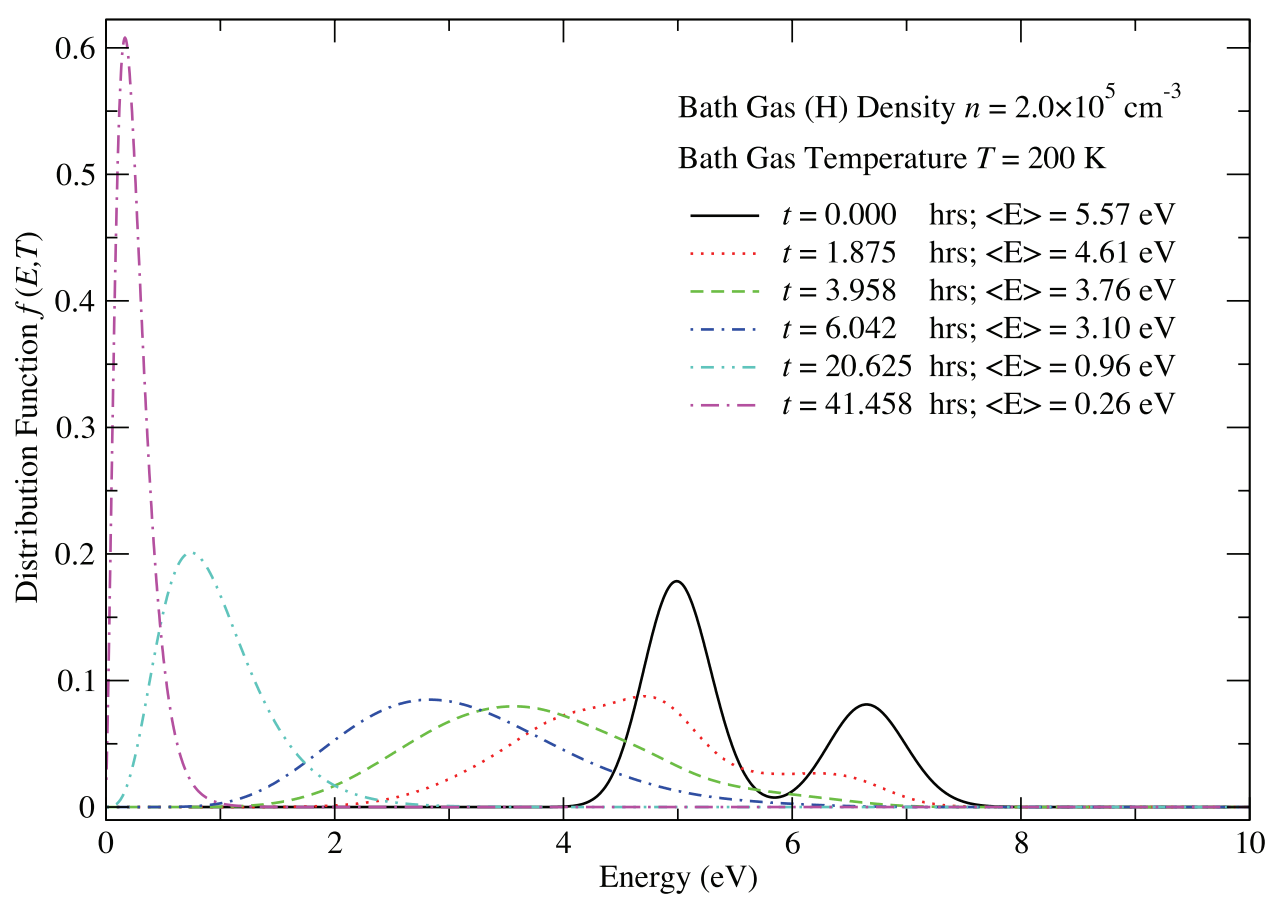

Figure 12. Energy distribution functions at selected relaxation times in hours of fast $\mathrm{O}$ atoms thermalized in $\mathrm{H}$ bath gas with temperature of $200 \mathrm{~K}$ and a density of $2.0 \times 10^{5} \mathrm{~cm}^{-3}$.

delays to reach the same energy measured in $k_{\mathrm{B}} T$ for bath gas temperatures of 400 and $1000 \mathrm{~K}$ with respect to $200 \mathrm{~K}$ are 43.9 and $97.5 \mathrm{~s}$. With hard-sphere collisions, the differences in the energy relaxation curve at $200 \mathrm{~K}$ come only from the change of cross sections if we scale the temperature ratio by $\sqrt{T_{2} / T_{1}}$ [Andersen and Shuler, 1964] and shift according to the corresponding time delays the energy relaxation curves at 400 and $1000 \mathrm{~K}$, where $T_{1}=200 \mathrm{~K}$ and $T_{2}=400$ or $1000 \mathrm{~K}$. The resulting curves are given in Figures $14 \mathrm{a}$ and $14 \mathrm{~b}$, respectively. If the cross sections were independent of the temperature, one would expect a full overlap of the relaxation curve of $200 \mathrm{~K}$ and the shifted curves at 400 and $1000 \mathrm{~K}$. However, in both cases, we see that this scaling shift leads to the slower relaxation, indicating that the effective hard-sphere cross sections are becoming smaller with the increase of the bath gas temperature. A relatively small temperature dependence was found for heavy particles relaxing in light bath gas, consistent with the results of energy loss rate derived from the Boltzmann kernel. The energy loss rate $\gamma(E, T)$ was obtained directly from the solution of the Boltzmann equation, and compared with that approximated through the integration of the kernel of the Boltzmann equation. In general, the agreement is good. Especially, during the longer relaxation time period, where the distributions of the projectile particles relax through continuous quasi-Maxwellian distributions, the agreement is better. The large deviation at the beginning of the relaxation reflects the fact that the distribution functions of the initial projectile particles are non-Maxwellian.

\section{Conclusion}

[32] The interaction potentials between $\mathrm{O}\left({ }^{3} P\right)$ and $\mathrm{H}\left({ }^{2} S\right)$ were constructed using multireference configuration interaction methods. The derived dissociation energy for the

Table 4. Effective Hard-Sphere Cross Sections $\sigma$ Fitted to the Solutions of Linear Boltzmann Equation for Different Energy Ranges ${ }^{\mathrm{a}}$

\begin{tabular}{|c|c|c|c|c|c|c|c|}
\hline \multirow[b]{2}{*}{ Projectile } & \multirow[b]{2}{*}{ Bath Gas } & \multicolumn{2}{|c|}{$200 \mathrm{~K}$} & \multicolumn{2}{|c|}{$400 \mathrm{~K}$} & \multicolumn{2}{|c|}{$1000 \mathrm{~K}$} \\
\hline & & Energy Range & $\sigma$ & Energy Range & $\sigma$ & Energy Range & $\sigma$ \\
\hline $\mathrm{O}$ & $\mathrm{H}$ & $5.0 \leq \mathrm{E}<11.0$ & $1.47 \times 10^{-15}$ & $5.0 \leq \mathrm{E}<11.0$ & $1.47 \times 10^{-15}$ & $1.5 \leq \mathrm{E}<11.0$ & $1.38 \times 10^{-15}$ \\
\hline $\mathrm{O}$ & $\mathrm{H}$ & $1.5 \leq \mathrm{E}<5.00$ & $1.70 \times 10^{-15}$ & $1.5 \leq \mathrm{E}<5.00$ & $1.60 \times 10^{-15}$ & $0.0<\mathrm{E}<1.50$ & $1.73 \times 10^{-15}$ \\
\hline $\mathrm{O}$ & $\mathrm{H}$ & $0.4 \leq \mathrm{E}<1.50$ & $2.12 \times 10^{-15}$ & $0.4 \leq \mathrm{E}<1.50$ & $2.00 \times 10^{-15}$ & & \\
\hline $\mathrm{O}$ & $\mathrm{H}$ & $0.0<\bar{E}<0.40$ & $2.45 \times 10^{-15}$ & $0.0<\mathrm{E}<0.40$ & $2.10 \times 10^{-15}$ & & \\
\hline $\mathrm{O}$ & D & $6.0 \leq \mathrm{E}<11.0$ & $1.10 \times 10^{-15}$ & $6.0 \leq \mathrm{E}<11.0$ & $1.08 \times 10^{-15}$ & $6.0 \leq \mathrm{E}<11.0$ & $1.07 \times 10^{-15}$ \\
\hline $\mathrm{O}$ & D & $0.8 \leq \mathrm{E}<6.00$ & $1.53 \times 10^{-15}$ & $0.8 \leq \mathrm{E}<6.00$ & $1.51 \times 10^{-15}$ & $0.8 \leq \mathrm{E}<6.00$ & $1.41 \times 10^{-15}$ \\
\hline $\mathrm{O}$ & $\mathrm{D}$ & $0.0<\mathrm{E}<0.80$ & $2.41 \times 10^{-15}$ & $0.0<\mathrm{E}<0.80$ & $2.10 \times 10^{-15}$ & $0.0<\mathrm{E}<0.80$ & $1.79 \times 10^{-15}$ \\
\hline $\mathrm{H}$ & $\mathrm{O}$ & $5.0 \leq \mathrm{E}<11.0$ & $0.40 \times 10^{-15}$ & $5.0 \leq \mathrm{E}<11.0$ & $0.30 \times 10^{-15}$ & $7.0 \leq \mathrm{E}<11.0$ & $0.26 \times 10^{-15}$ \\
\hline $\mathrm{H}$ & $\mathrm{O}$ & $1.0 \leq \mathrm{E}<5.00$ & $0.92 \times 10^{-15}$ & $1.0 \leq \mathrm{E}<5.00$ & $0.85 \times 10^{-15}$ & $1.0 \leq \mathrm{E}<7.00$ & $0.67 \times 10^{-15}$ \\
\hline $\mathrm{H}$ & $\mathrm{O}$ & $0.4 \leq \mathrm{E}<1.00$ & $1.31 \times 10^{-15}$ & $0.4 \leq \mathrm{E}<1.00$ & $1.25 \times 10^{-15}$ & $0.0<\mathrm{E}<1.00$ & $1.92 \times 10^{-15}$ \\
\hline $\mathrm{H}$ & $\mathrm{O}$ & $0.0<\mathrm{E}<0.40$ & $2.30 \times 10^{-15}$ & $0.0<\mathrm{E}<0.40$ & $2.20 \times 10^{-15}$ & & \\
\hline D & $\mathrm{O}$ & $5.0 \leq \mathrm{E}<11.0$ & $0.31 \times 10^{-15}$ & $5.0 \leq \mathrm{E}<11.0$ & $0.31 \times 10^{-15}$ & $5.0 \leq \mathrm{E}<11.0$ & $0.32 \times 10^{-15}$ \\
\hline $\mathrm{D}$ & $\mathrm{O}$ & $0.2 \leq \mathrm{E}<5.00$ & $1.11 \times 10^{-15}$ & $0.4 \leq \mathrm{E}<5.00$ & $0.99 \times 10^{-15}$ & $1.0 \leq \mathrm{E}<5.00$ & $0.87 \times 10^{-15}$ \\
\hline D & $\mathrm{O}$ & $0.0<\mathrm{E}<0.20$ & $2.82 \times 10^{-15}$ & $0.0<\mathrm{E}<0.40$ & $2.40 \times 10^{-15}$ & $0.0<\mathrm{E}<1.00$ & $1.90 \times 10^{--15}$ \\
\hline
\end{tabular}

${ }^{\mathrm{a}}$ Hard-sphere cross sections $\sigma$ given in $\mathrm{cm}^{2}$ and energy ranges given in $\mathrm{eV}$. 


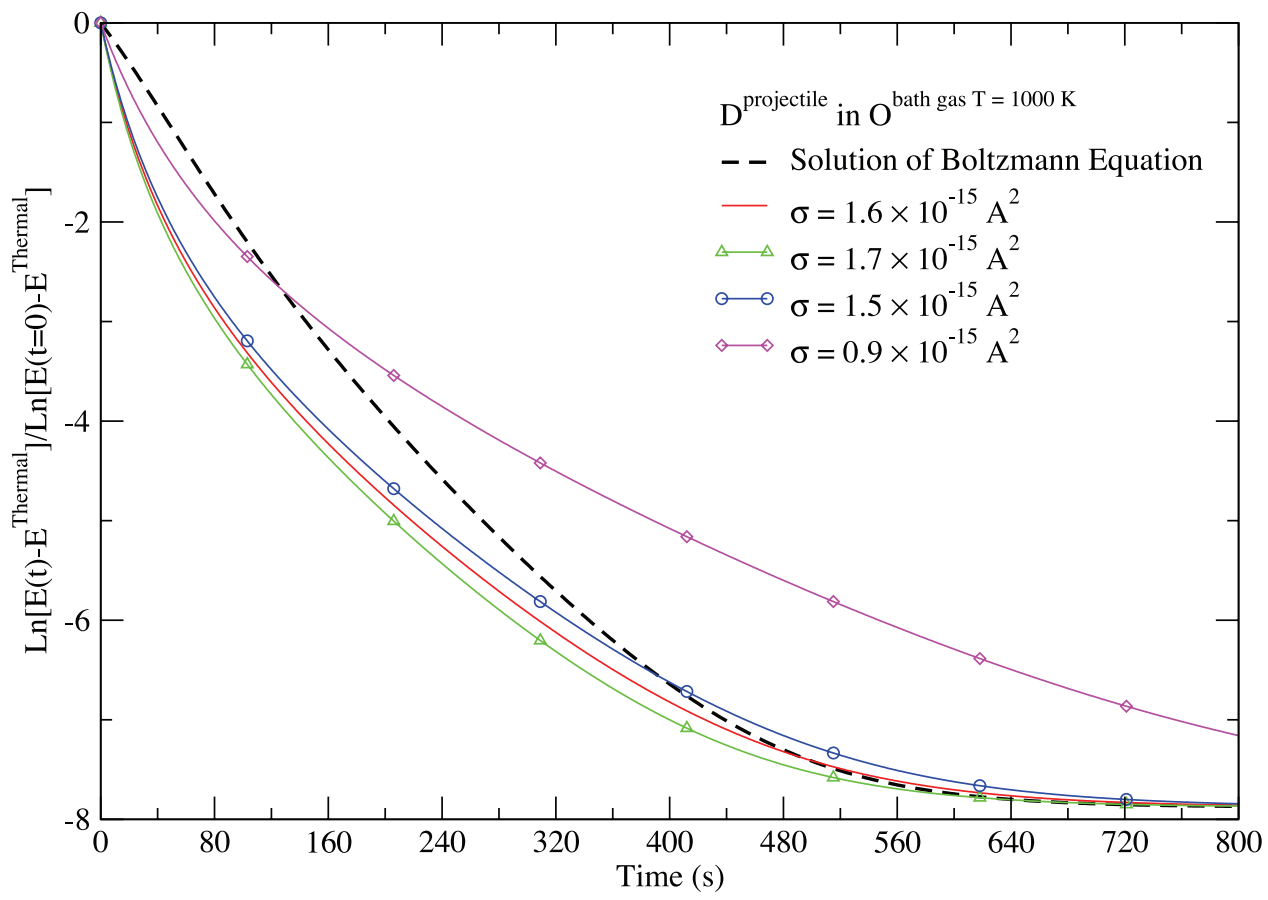

Figure 13. Translational energy relaxation versus time in seconds for fast $\mathrm{D}$ atoms thermalized in $\mathrm{O}$ bath gas with temperature of $200 \mathrm{~K}$. The dashed line is obtained from the solution of linear Boltzmann kinetic equations, and the solid lines with symbols are determined using the hard-sphere approximation with different hard-sphere radii.
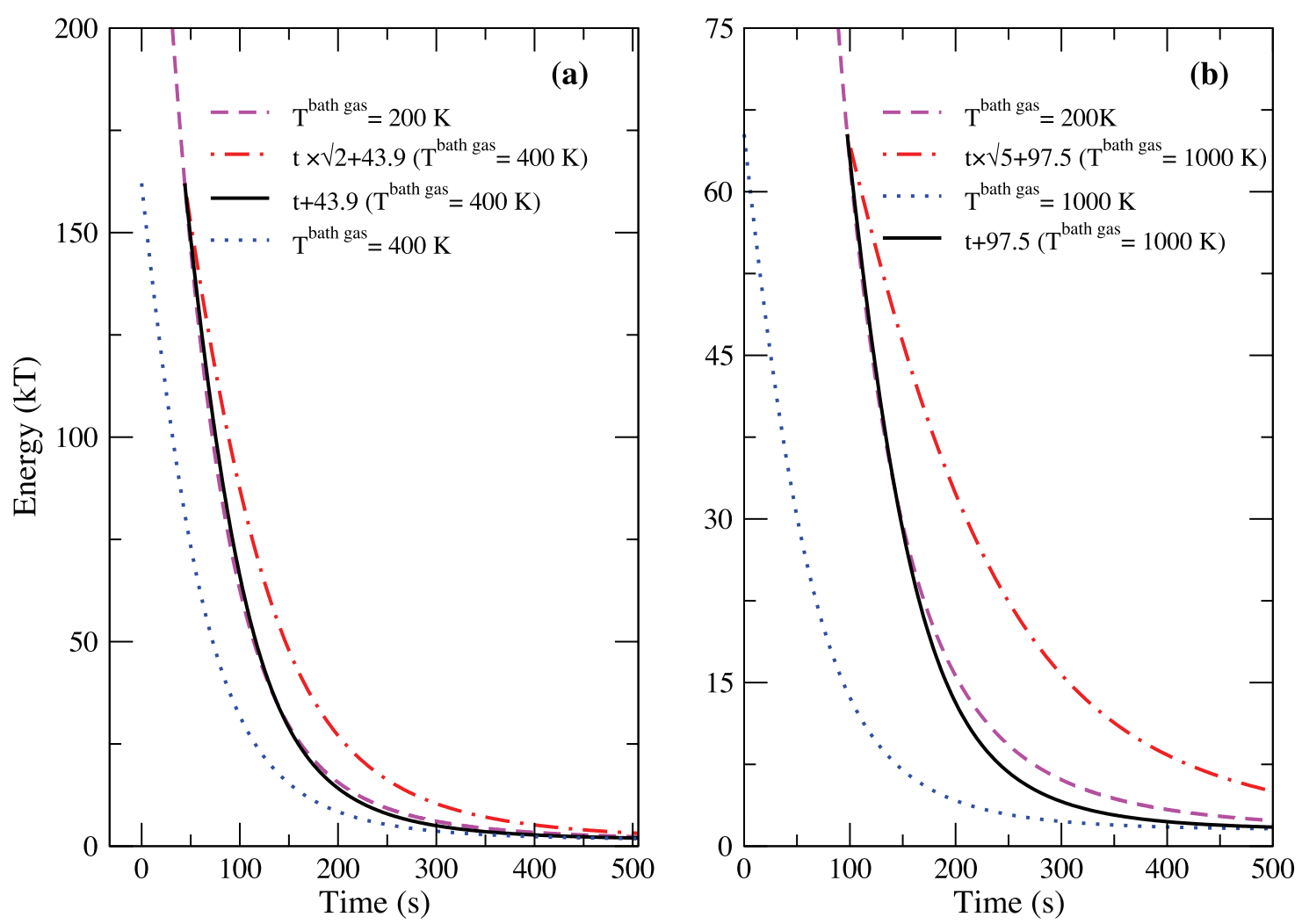

Figure 14. Translational energy relaxation versus time in seconds for fast $\mathrm{H}$ atoms thermalized in $\mathrm{O}$ bath gas with temperatures of 200,400 , and $1000 \mathrm{~K}$. The scaling of the relaxation curves at different temperatures according to $\sqrt{T_{2} / T_{1}}$ shows the temperature dependence of the effective hard-sphere cross section. 
$\tilde{X}^{2} \Pi$ state agrees closely with experimental data. On the basis of the new potentials, elastic collisions of $\mathrm{O}$ with $\mathrm{H}$ and its isotope $\mathrm{D}$ were studied quantum mechanically in the energy range up to $10 \mathrm{eV}$. The results are consistent with our previous calculations, and validate both. The structure of the differential cross sections was analyzed using the classical scattering theory. The agreement at intermediate energies between the quantal and quasi-classical calculations establishes the validity of quasi-classical theory for the description of the collision process at higher energies.

[33] The relaxation kinetics of energetic $\mathrm{O}$ or $\mathrm{H}(\mathrm{D})$ atoms in the $\mathrm{H}(\mathrm{D})$ or $\mathrm{O}$ bath gases corresponding to different atmospheric conditions were examined through the numerical solution of the linear Boltzmann equation where the kernel of the Boltzmann equation was constructed from quantal differential cross sections. The collision frequency, energy loss rate, the distribution of secondary recoil energetic atoms and their temperature and mass dependence were investigated. For actual applications to planetary atmosphere, effective hard-sphere cross sections that closely reproduce the energy relaxation kinetics are provided.

[34] Acknowledgments. The research of A.D. and P.Z. was supported by the National Science Foundation under grant ATM-0430506, and the research of V.K. was supported by NASA grants NNX07AR03G and NNG06GD40G. The computational resource was provided by the National Center for Atmospheric Research (NCAR).

[35] Wolfgang Baumjohann thanks James Duff and another reviewer for their assistance in evaluating this paper.

\section{References}

Andersen, K., and K. E. Shuler (1964), On the relaxation of the hard-sphere Rayleigh and Lorentz gas, J. Chem. Phys., 40, 633, doi:10.1063/ 1.1725183 .

Chapman, S, and T. G. Cowling (1939), The Mathematical Theory of NonUniform Gases, Cambridge Univ. Press, London.

Colbert, D. T., and W. H. Miller (1992), A novel discrete variable representation (DVR) for quantum mechanical reactive scattering via the Smatrix Kohn method, J. Chem. Phys., 96, 1982, doi:10.1063/1.462100.

Cooper, D. L., J. H. Yee, and A. Dalgarno (1984), Energy transfer in oxygen-hydrogen collisions, Planet. Space Sci., 32, 825, doi:10.1016/ 0032-0633(84)90005-9.

Davidson, E. R, and D. W. Silver (1977), Size consistency in the dilute helium gas electronic structure, Chem. Phys. Lett., 52, 403, doi:10.1016/ 0009-2614(77)80475-2.

Esposti, A. D., and H.-J. Werner (1990), Ab initio calculation of the $\mathrm{OH}$ $\left(X^{2} \Pi, \mathrm{A}^{2} \Sigma^{+}\right)+$Ar potential energy surfaces and quantum scattering studies of rotational energy transfer in the $\mathrm{OH}\left(\mathrm{A}^{2} \Sigma^{+}\right)$state, J. Chem. Phys., 93, 3351, doi:10.1063/1.458816.

Forrey, R. C., L. You, V. Kharchenko, and A. Dalgarno (1996), Index of refraction of noble gases for sodium matter waves, Phys. Rev. A, 54, 2180, doi:10.1103/PhysRevA.54.2180.

Guberman, S. L (1988), The production of O (1D) from dissociative recombination of $\mathrm{O}_{2}+$, Planet. Space Sci., 36, 47, doi:10.1016/00320633(88)90145-6.

Gurwell, M. A, and Y. L. Yung (1993), Fractionation of hydrogen and deuterium on Venus due to collisional ejection, Planet. Space Sci., 41, 91, doi:10.1016/0032-0633(93)90037-3.

Hodges, R. R (1993), Collision cross sections and diffusion parameters for $\mathrm{H}$ and $\mathrm{D}$ in atomic oxygen, J. Geophys. Res., 98, 3799, doi:10.1029/ 92JA02606.

Huber, K. P, and G. Herzberg (1979), Molecular Spectra and Molecular Constants IV: Constants of Diatomic Molecules, Van Nostrand, New York.
Jamieson, M. J., A. Dalgarno, and L. Wei (2006), Elastic scattering of hydrogen and deuterium atoms by oxygen atoms, J. Geophys. Res., 111, A06308, doi:10.1029/2005JA011361.

Joens, J. A. (2001), The dissociation energy of $\mathrm{OH}\left(X^{2} \Pi\right)$ and the enthalpy of formation of $\mathrm{OH}\left(X^{2} \Pi\right), \mathrm{ClOH}$ and $\mathrm{BrOH}$ from thermochenical cycles, J. Phys. Chem. A, 105, 11041, doi:10.1021/jp011833u.

Kharchenko, V, and A. Dalgarno (2004), Thermalization of fast O ( $\left.{ }^{1} \mathrm{D}\right)$ atoms in the stratosphere and mesosphere, J. Geophys. Res., 109, D18311, doi:10.1029/2004JD004597.

Kharchenko, V., J. Tharamel, and A. Dalgarno (1997), Kinetics of thermalization of fast nitrogen atoms beyond the hard sphere approximation, J. Atoms. Sol. Terr. Phys., 59, 107.

Kharchenko, V., N. Balakrishnan, and A. Dalgarno (1998), Thermalization of fast nitrogen atoms in elastic and inelastic collisions with molecules of atmospheric gases, J. Atoms. Sol. Terr. Phys., 60, 95.

Kharchenko, V., A. Dalgarno, B. Zygelman, and J.-H. Yee (2000), Energy transfer in collisions of oxygen atoms in the terrestrial atmosphere, J. Geophys. Res., 105, 24899, doi:10.1029/2000JA000085.

Knowles, P. J., and H.-J. Werner (1988), An efficient method for the evaluation of coupling coefficients in configuration interaction calculations, Chem. Phys. Lett., 145, 514, doi:10.1016/0009-2614(88)87412-8.

Krest'yanikovam, M. A., and V. I. Shematovich (2005), Stochastic Models of Hot Planetary and Satellite Coronas: A Photochemical Source of Hot Oxygen in the Upper Atmosphere of Mars, Sol. Syst. Res. Engl. Transl., $39,26$.

Landau, L. D., and L. M. Lifshitz (1991), Quantum Mechanics: NonRelativistic Theory, 3rd ed., Butterworth-Heinemann, Oxford, U. K.

McElroy, M. B., M. J. Prather, and J. M. Rodriguez (1982), Escape of hydrogen from Venus, Science, 215, 1614, doi:10.1126/ science.215.4540.1614.

Mott, N. F, and H. S. W. Massey (1965), The Theory of Atomic Collisions, Oxford Univ. Press, New York.

Oppenheim, I., K. Schuler, and G. Weiss (1977), Stochastic Processes in Chemical Physics: The Master Equation, MIT Press, Cambridge, Mass.

Ruscic, B., et al. (2002), On the enthalpy of formation of hydroxyl radical and gas-phase bond dissociation energies of water and hydroxyl, J. Phys. Chem. A, 106, 2727, doi:10.1021/jp013909s.

Shizgal, B. D (1998), An analysis of O-H interaction potential, O-H and O-D collision cross sections, and vibrational states, Planet. Space Sci., 47, 163, doi:10.1016/S0032-0633(98)00088-9.

Shizgal, B. D. (1999), Escape of H and D from Mars and Venus by energization with hot oxygen, J. Geophys. Res., 104, 14833, doi:10.1029/ 1999JA900157.

Shizgal, B. D., and G. G. Arkos (1996), Nonthermal escape of the atmospheres of Venus, Earth, and Mars, Rev. Geophys., 34, 483, doi:10.1029/ 96RG02213.

Werner, H.-J., and P. J. Knowles (1988), An efficient internally contracted multiconfiguration-Reference configuration interaction method, J. Chem. Phys., 89, 5803, doi:10.1063/1.455556.

Wilson, A. K., T. V. Mourik, and T. H. Dunning (1996), Gaussian basis sets for use in correlated molecular calculations. Part VI. Sextuple zeta correlation consistent basis sets for boron through neon, J. Mol. Struct., 388, 339.

Woon, D. E., and T. H. Dunning (1994), Gaussian basis sets for use in correlated molecular calculations. Part IV. Calculation of static electrical response properties, J. Chem. Phys., 100(4), 2975, doi:10.1063/1.466439.

Yarkony, D. R. (1992), A theoretical treatment of the predissociation of the individual rovibronic levels of $\mathrm{OH} / \mathrm{OD}\left(\mathrm{A}^{2} \Sigma^{+}\right)$, J. Chem. Phys., 97, 1838, doi:10.1063/1.463172.

Zhang, P., V. Kharchenko, and A. Dalgarno (2007), Thermalization of suprathermal N ( $\left.{ }^{4} \mathrm{~S}\right)$ atoms in He and Ar gases, Mol. Phys., 105, 1487, doi:10.1080/00268970701210008.

Zhang, P, V. Kharchenko, A. Dalgarno, Y. Matsumi, T. Nakayama, and K. Takahashi (2008), Approach to thermal equilibrium in atomic collision, Phys. Rev. Lett., 100, 103001, doi:10.1103/PhysRevLett.100. 103001 .

A. Dalgarno, V. Kharchenko, and P. Zhang, Institute for Theoretical Atomic, Molecular and Optical Physics, Harvard-Smithsonian Center for Astrophysics, 60 Garden Street, Mail Stop 14, Cambridge, MA 02138, USA. (pezhang@cfa.harvard.edu)

M. J. Jamieson, Department of Computing Science, University of Glasgow, Sir Alwyn Williams Building, Glasgow G12 8QQ, UK. 